\title{
The Spitzer View of the Extragalactic Universe*
}

\author{
Baruch T. Soifer, ${ }^{1}$ George Helou, ${ }^{1}$ \\ and Michael Werner ${ }^{2}$
}

\footnotetext{
${ }^{1}$ Division of Physics, Mathematics and Astronomy, California Institute of Technology, Pasadena, California 91125; email: bts@ipac.caltech.edu, gxh@ipac.caltech.edu

${ }^{2}$ Astronomy and Physics Directorate, Jet Propulsion Laboratory, California Institute of Technology, Pasadena, California 91109; email: mwerner@sirtfweb.jpl.nasa.gov
}

Annu. Rev. Astron. Astrophys. 2008. 46:201-40

The Annual Review of Astronomy and Astrophysics is online at astro.annualreviews.org

This article's doi:

10.1146/annurev.astro.46.060407.145144

Copyright (c) 2008 by Annual Reviews.

All rights reserved

0066-4146/08/0922-0201\$20.00

*The U.S. Government has the right to retain a nonexclusive, royalty-free license in and to any copyright covering this paper.

\section{Key Words}

aromatic emission bands, far-infrared background, infrared astronomy, massive high-redshift galaxies, star formation history, ultraluminous infrared galaxies

\begin{abstract}
The Spitzer Space Telescope was launched in August 2003. Scientists from around the world have applied its orders-of-magnitude gain in imaging and spectroscopic capability to a wide array of topics in extragalactic research. Spitzer studies have found massive galaxies at redshifts greater than 6, resolved the cosmic background at $200 \mu \mathrm{m}>\lambda>20 \mu \mathrm{m}$ into the dusty infraredluminous galaxies that comprise it, directly detected dust-enshrouded star formation, and measured the star formation history of the universe to $z>3$. In this review we examine a small fraction of the extragalactic studies from Spitzer that have been conducted in its first three years of operations.
\end{abstract}




\section{INTRODUCTION AND BACKGROUND}

The launch of the Spitzer Space Telescope opened a new era for infrared (IR) astronomy. Its vast leap in sensitivity over previous platforms, both space and ground, has enabled Spitzer to address virtually every aspect of observational astronomy. This review, a companion article to that describing the early Spitzer results in galactic and solar system astronomy (Werner et al. 2006), addresses Spitzerbased contributions to date in extragalactic astronomy. It is already impossible within the confines of one ARAA review to summarize all of Spitzer's contributions in this field, and we have been forced to severely limit the topics covered. We have chosen to discuss those areas that we believe the Spitzer contributions have made the greatest impact in extragalactic research.

Work from prior IR space missions in the general area of extragalactic astronomy has been reviewed in these volumes [Infrared Astronomical Satellite (IRAS), Soifer et al. 1987; Infrared Space Observatory (ISO), Genzel \& Cesarsky 2000]. Werner et al. give a general overview of the Spitzer Observatory and we refer the reader to that description, or a more detailed one in Gehrz et al. (2007). This review includes papers submitted for publication through fall 2007, more than four years since launch. As of this writing, the cryogenic mission is expected to last until mid-April 2009. A warm Spitzer mission, entirely dedicated to the two shortest-wavelength bands and described below, will follow the completion of the cryogenic mission.

In Section 2, we describe the progress in determining the star-formation history of the universe, from the local universe to beyond $z \sim 6$. Section 3 reports the advances in our understanding of the far-IR $(\lambda>20 \mu \mathrm{m})$ universe, and Section 4 describes advances in our understanding of the phenomenology and physics of individual classes of extragalactic objects. Finally, in Section 5 we summarize the key results to date and future directions for work with Spitzer and other facilities.

\section{THE STAR-FORMATION HISTORY OF THE UNIVERSE}

\subsection{Introduction}

The determination of the history of star formation in the universe has been a major thrust of observational cosmology and galaxy evolution studies for decades. Beginning with the groundbreaking work of Lilly et al. (1995) and Madau et al. (1996), workers derived the star-formation rate (SFR) as a function of lookback time using optical and UV observations of galaxies. It was quickly realized that such determinations were dominated by the effects of dust extinction on the brightness of galaxies in the rest-frame UV. Corrections to apparent UV luminosities, based on the UV and IR properties of galaxies in the local universe (Meurer, Heckman \& Calzetti 1999), are in the range of 4-7.

With Spitzer data the general approach is to use the 24- $\mu \mathrm{m}$ measurements of galaxies (with the relative sensitivities of 24-, 70-, and 160- $\mu \mathrm{m}$ Spitzer observations, $24 \mu \mathrm{m}$ is by far the most sensitive channel for detecting distant galaxies) along with a bolometric correction to determine total IR $(8-1000 \mu \mathrm{m})$ luminosities. [The bolometric correction depends on the galaxy's redshift, and its uncertainty increases with redshift (Chary \& Elbaz 2001, Xu et al. 2001, Dale \& Helou 2002, Elbaz et al. 2002). At $z=2$, for example, the bolometric correction factor, i.e., $\mathrm{L}_{\mathrm{bol}} / v \mathrm{~L}_{\mathrm{v}}$ (24 $\mu \mathrm{m}$, obs), is $\sim 5-30$.] The emission at $\lambda>8 \mu \mathrm{m}$ is partly or mostly attributed to the dustobscured star formation in the galaxy, and a contribution from the observed UV radiation is included. The luminosity in newly formed stars, dominated by the most luminous (massive) stars in the galaxy, is converted to an SFR through a simple relation $\dot{M}=K L$ (star formation). [L(star formation) refers to the luminosity associated with newly formed stars. Most workers use $K=1.7 \times$ $10^{-10}$ (where $\dot{M}$ is in solar masses per year and $L$ is in solar bolometric luminosity units) from Kennicutt (1998). This factor has embedded the assumption that the initial mass function (IMF) 
follows the Salpeter law, $\mathrm{N}(M) \sim M_{*}^{-2.35}$, between 0.1 and $100 M_{\odot}$. This is a source of substantial uncertainty, particularly in actively forming galaxies where other IMFs have been suggested that have fewer stars with $M<1 M_{\odot}$ (e.g., Kroupa 2001, Chabrier 2003).]

Another key parameter is the specific star-formation rate (SSFR), which is the SFR described above divided by the galaxy's stellar mass $\left(M_{*}\right)$. The stellar mass of a galaxy is generally determined by fitting models of star formation histories to the observed multiwavelength spectral energy distribution (SED).

SED fitting is fraught with great uncertainties in determining physical parameters such as galaxy mass, because several key parameters (e.g., dust extinction, metallicity, star-formation history) can be traded off to generate quite similar SEDs. Often the redshift of the system (photo-z) is being fitted as well, which adds yet another degree of freedom. Another substantial uncertainty in derived galaxy mass has recently arisen regarding the contribution from the thermal pulsation phase of asymptotic giant branch (AGB) stars (TP-AGB, Maraston 2005, Maraston et al. 2006). The galaxy masses derived by Maraston et al. are substantially (>50\%) lower than those based on older models.

Results have fewer uncertainties when more wavelengths are available for the SED fitting. Fields where a large amount of multispectral data is available (see sidebar, Fields of Dreams) are heavily analyzed and provide samples of many thousands of galaxies in reasonably fine-grained redshift bins, even with the use of photometric redshifts. Those fields where multiwavelength data are less complete have concomitantly higher uncertainties in photo-z's and other derived parameters. The existence of large samples of galaxies over wide redshift ranges, where the analysis is done in a consistent manner (e.g., assumed star-formation history, metallicity, etc.), gives confidence that even if there are large uncertainties (factors of three or more) in derived absolute numbers, the variations of parameters (e.g., SFR, SSFR) with redshift are being measured correctly.

With this as a cautionary note of introduction, we review the investigations of the star-forming history of the universe enabled by Spitzer observations.

\subsection{The Local Universe $(z<2)$}

Many early Spitzer studies of star formation in the local universe focused on the Chandra Deep Field-South/Galaxy Evolution from Morphology and Spectral Energy Distributions (CDFS/GEMS) field because of its wealth of multispectral data. Pérez-González et al. (2005) and Le Floc'h et al. (2005) focused on a broad range of redshifts for sources in the CDF-S/GEMS field and the Hubble Deep Field-North (HDF-N) field (Pérez-González et al. 2005). These investigations predominantly utilized photometric redshifts to select their $z$ bins.

Pérez-González et al. and LeFloc'h et al. derive the SFR history from $24-\mu \mathrm{m}$ surveys by establishing the IR luminosity function as a function of redshift, integrating this function to determine the IR luminosity per $\mathrm{Mpc}^{3}$ and converting this to a SFR per $\mathrm{Mpc}^{3}$ using the relation between SFR and luminosity. These results agree well and find an increase in the IR luminosity density and, hence, SFR per $\mathrm{Mpc}^{3}$ by an order of magnitude between $z=0$ and $z \sim 1$. PérezGonzález et al. find that the SFR per $\mathrm{Mpc}^{3}$ continues to increase to $z \sim 1.5$, and then flattens to $z \sim 3$. These works are consistent with the UV-based SFR versus $z$ plots (Lilly-Madau diagrams) where the UV-based analyses apply the large corrections for dust extinction described above. Figure 1 shows the IR luminosity density (star-formation rate) versus redshift from these works as well as work (discussed below) that pushes the determinations to still higher redshifts. Bai et al. (2007) and Marcillac et al. (2007) find similar evolution with redshift in cluster galaxies.

LeFloc'h et al. break down the contribution to the IR luminosity into lower-luminosity systems $\left(L_{\mathrm{ir}}<10^{11} L_{\odot}\right)$, luminous IR galaxies (LIRGs, $\left.10^{11} L_{\odot}<L_{\mathrm{ir}}<10^{12} L_{\odot}\right)$ and ultraluminous IR galaxies (ULIRGs, $L_{\mathrm{ir}}>10^{12} L_{\odot}$ ). For $z<0.7$, they find that lower-luminosity systems dominate, 


\section{FIELDS OF DREAMS}

Much of the extragalactic work described in this review results from Spitzer surveys of areas of the sky ranging in extent from tens of square arcminutes to tens of square degrees. Most of these surveys cover areas that are wellstudied with many other observatories, thereby producing a unique multiwavelength view of the universe. We list here the salient properties of the Legacy imaging survey programs that Spitzer has conducted or will conduct through observing Cycle 4. See the URLs provided for information on the other data sets available. The surveys (apart from SINGS/LVLS) are listed in approximate order of increasing depth of the Spitzer observations. Other major imaging surveys have been conducted as part of Guaranteed Time Observer programs. Numerous spectroscopic surveys have contributed essential results to this review, but are not listed here.

SWIRE. The Spitzer Wide-Area Infrared Extragalactic Survey (Cycle 0, http://swire.ipac.caltech.edu/ swire/swire.html) 50 square degrees, six separate areas, MIPS and IRAC.

FLS. The First Look Survey (extragalactic component) (http://ssc.spitzer.caltech.edu/fls/extragal/) 4 square degrees, MIPS and IRAC.

SDWFS. The Spitzer Deep, Wide-Field Survey in the NOAO Deep Wide-Field Survey Boötes area (http:// www.noao.edu/noao/noaodeep/). (Cycle 4) the NDWFS "Boötes" field—about 8.5 square degrees—IRAC (following major GTO programs using IRAC and MIPS).

S-COSMOS. (Cycle 2, 3) 2-square-degree COSMOS field, IRAC, and MIPS (http://www.ifa.hawaii.edu/ 〜ilbert/S-Cosmos/).

UKIDSS. (Cycle 4) 1-square-degree field UKIDSS Ultra Deep Survey (http://www.ukidss.org/surveys/ surveys.html), IRAC, and MIPS imaging survey.

SIMPLE. (Cycle 2) 1/4 square degree around the Chandra Deep Field South (http://www.astro.yale.edu/ dokkum/simple/) IRAC.

GOODS/FIDEL. The Great Observatories Origins Deep Survey (Cycle 0, http://www.stsci.edu/science/ goods) 320 square arcmin centered on the HDF-N and the CDF-S fields. IRAC and MIPS 24- $\mu$ m imaging. Spitzer's deepest observations have been made over portions of the GOODS field. The FIDEL project (Cycle 3) in part extends this imaging to $70 \mu \mathrm{m}$.

SINGS/LVLS. The Spitzer Infrared Nearby Galaxies Survey (Cycle 0, http://sings.stsci.edu/) imaging and spectroscopic study of 75 nearby galaxies IRAC, MIPS, IRS. LVLS (Cycle 4) extends IRAC and MIPS imaging to a volume complete sample of 258 galaxies within $11 \mathrm{Mpc}$.

whereas for $z>0.7$, LIRGs dominate the energy production, producing $\sim 70 \%$ of the total IR energy density at $z \sim 1$. The contribution from ULIRGs is rising steeply with redshift, being negligible ( $<3 \%$ of the total) at $z<0.3$ and $\sim 20 \%$ of the energy at $z \sim 1$. Analyses by Babbedge et al. (2006) and Zheng et al. (2006) show excellent agreement with these results. Caputi et al. (2007) find an IR luminosity density at $z=1$ of $1.2 \times 10^{9} \mathrm{~L}_{\odot} \mathrm{Mpc}^{-3}$, corresponding to an SFR of $0.2 M_{\odot}$ year $^{-1} \mathrm{Mpc}^{3}$.

Noeske et al. $(2007 \mathrm{a}, \mathrm{b})$ analyze the SFR as a function of redshift in the Extended Groth Strip (EGS). Using the 24- $\mu$ m fluxes and optical emission line spectroscopy to determine SFRs, they find a clear band of SFRs as a function of galaxy mass referred to as a "main sequence" of star formation in galaxies, which, at a given redshift, increases with galaxy mass. The SFR increases by a factor of three from redshift $z=0.36$ to $z=1$, consistent with the results of Pérez-González et al. and LeFloc'h et al. while the slope of the SFR versus $M *$ main sequence appears to be independent of redshift. Zheng et al. (2007) and Noeske et al. (2007a) find that the SSFR decreases with $M_{*}$, and the observed star-formation episode is the dominant one in assembling the stellar mass for the lower-mass galaxies with high SSFRs. Noeske et al. suggest that lower-mass galaxies experience 


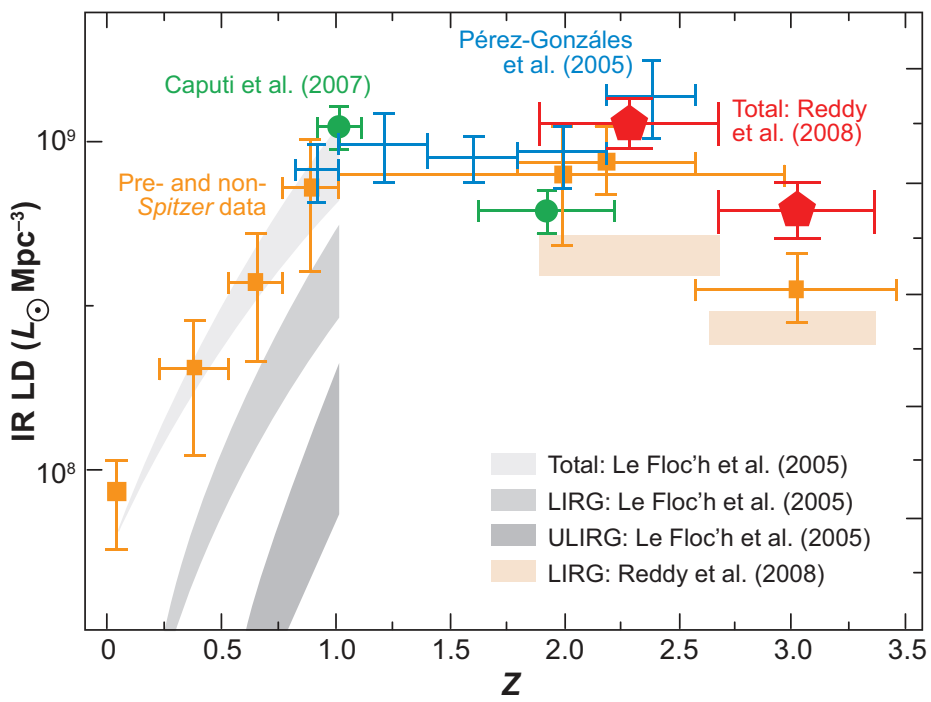

Figure 1

The infrared luminosity density (IR LD) as a function of redshift $z$ to $z=3.5$ (taken from Reddy et al. 2008, used by permission). The orange points represent pre- and non-Spitzer data. The Spitzer-based data include results from Reddy et al. (2008), Caputi et al. (2007), Pérez-González et al. (2005), and Le Floc'h et al. (2005). The bands indicate the total, LIRG, and ULIRG contribution to the total IR LD at $z<1$, as determined by Le Floc'h et al.

this dominant star-forming episode with a later onset and a longer decay timescale than do more massive galaxies.

Bell et al. (2007) find that in the redshift range of 0.3-0.9 the bulk (80-85\%) of the star formation occurs in "blue cloud" galaxies. They find consistency between the global SFR and the growth of stellar mass over this redshift range and conclude that a large fraction of blue galaxies must have their star formation quenched in some way to join the red sequence galaxies at lower redshifts.

\subsection{The More Distant Universe $(2<z<4)$}

Beginning with the pioneering work of Steidel and collaborators (Steidel \& Hamilton 1993, Steidel et al. 1996), large populations of galaxies have been identified and studied at redshifts ranging from $\sim 1.5-4$. Because of the extraordinary sensitivity of the Spitzer telescope (as deep as $0.1 \mu \mathrm{Jy}$, or AB magnitude 26.3 at $3.6 \mu \mathrm{m}$ ), Spitzer observations have become integral to these studies.

In the redshift range $2<z<4$, galaxies are readily detected in visible and near-IR bands out to $2.2 \mu \mathrm{m}$. Spitzer sensitivities are a very good match to these studies, and so extend the wavelength coverage to $8 \mu \mathrm{m}$, and in many cases distant galaxies are detected at $24 \mu \mathrm{m}$. At $z \sim 3$, IRAC observations correspond to rest-frame 1-2 $\mu \mathrm{m}$, wavelengths where late-type giants can dominate the stellar output of galaxies. The 1.6- $\mu \mathrm{m} \mathrm{H}$-opacity minimum provides a broad SED feature that is exploited to provide an independent photometric redshift estimator. The major theme of these studies has been to model galaxy SEDs in terms of star formation history and stellar mass, and to determine the mass contained in stars as a function of redshift. Recently, 24- $\mu \mathrm{m}$ observations have been added, predominantly in the GOODS fields, to provide direct determinations of IR luminosities (and, hence, dust-obscured SFRs). 
2.3.1. Spitzer studies of Lyman-break galaxies. Shapley et al. (2005) studied in detail 72 spectroscopically identified LBGs with $\langle z\rangle=2.3$ in the field of the quasar HS1700+6416. [LBGs were originally found photometrically by Steidel \& Hamilton (1993), and the spectroscopic confirmation of the technique was demonstrated by Steidel et al. (1996) and extended by Steidel et al. (1999) and Erb et al. (2003).] This study found that combining IRAC observations with shorter-wavelength observations did not resolve the degeneracies between age, dust content, and SFR in fitting models to the observed SEDs. They find that the stellar masses derived using IRAC data agree with those derived previously with a factor of five smaller dispersion in the observed mass/luminosity $(M / L)$ ratio at $4.5 \mu \mathrm{m}$ as compared to those using only shorter-wavelength data (even with the inclusion of IRAC data, the range in the derived $M / L$ is still a factor of $15-$ whether this is simply the range of acceptable model fits or is real variation in physical properties of galaxies is unclear). For this sample with $z \sim 2.3 \pm 0.3$, they find characteristic masses of Log $\left(M / M_{\odot}\right) \sim 10.3 \pm 0.5$. Shapley et al. conclude that the use of IRAC photometry reduces the uncertainties in derived mass by a factor of $1.5-2$, and that the average $M / L$ at $\mathrm{K}_{\mathrm{s}}$ and $4.5 \mu \mathrm{m}$ ( $R$ and $1.4 \mu \mathrm{m}$ in the rest-frame) are lower by factors of 5 and 2.5 , respectively, than local values.

2.3.2. Red galaxies. Distant red galaxies (DRGs) were defined by Franx et al. (2003) as having $\mathrm{J}_{-} \mathrm{K}_{\mathrm{s}}($ Vega $)>2.3 \mathrm{mag}(>1.37 \mathrm{AB})$ to place the Balmer/4000- $\AA$ break between the $\mathrm{J}$ and $\mathrm{K}_{\mathrm{s}}$ bands and thereby to identify systems in the range $2<z<3.5$ with reduced sensitivity to extinction by dust. Papovich et al. (2006) and Webb et al. (2006) have investigated DRGs utilizing IRAC and MIPS 24- $\mu \mathrm{m}$ data along with extensive additional data sets.

In the CDF-S GOODS field, Papovich et al. matched 132/153 DRGs (K selected sample with $\mathrm{K}_{\mathrm{s}}<24.1, \mathrm{~J}-\mathrm{K}_{\mathrm{s}}>2.3$ ) with IRAC detections; the rest of the DRGs are ruled out of having IRAC detections because of crowding. Of the DRGs in the CDF-S GOODS sample, 12 have spectroscopic redshifts; for the rest, photo- $z$ redshifts were estimated from SED fitting. In total, the median redshift of the sample is $\langle z\rangle=2.2$, in agreement with the sample of Franx et al. (2003). Half of the 153 DRGs in the CDF-S sample are associated with $24-\mu \mathrm{m}$ sources detected at $50 \mu \mathrm{Jy}$ or brighter.

Based on the SED fitting, $10 \%$ of the sample have best-fit models with ages $>1$ Gyr, suggesting passively evolving stellar populations. The vast majority have reddened stellar populations, and are split roughly equally between systems with large numbers of evolved stars (and a small amount of ongoing star formation) and heavily extincted starbursts. The luminosities suggested from the 24- $\mu \mathrm{m}$ detections are quite large: More than half of the DRGs with $24-\mu \mathrm{m}$ detections have IR luminosities greater than $10^{12} L_{\odot}$. Papovich et al. find the IR/UV ratios (i.e., IR luminosities, hence total SFRs) of DRGs agree with the $I R / U V$ versus $U V$ spectral slope relationship for local LIRGs (Goldader et al. 2002), but are more than an order of magnitude greater than predicted from nearby UV-starbursts (Meurer, Heckman \& Calzetti 1999).

Using multiwavelength photometry and Salpeter IMFs, Papovich et al. find similar stellar masses whether they use one or two ages of stellar populations in their fitting, with median masses in the range of $1.1-1.7 \times 10^{11} M_{\odot}$. Based on the derived masses, the global mass density of DRGs is $\sim 2.5 \times 10^{7} \mathrm{M}_{\odot} \mathrm{Mpc}^{-3}$ in the range of $2<z<3$.

For the DRGs detected at $24 \mu \mathrm{m}$, Papovich et al. find a mean SFR of $\sim 400 \mathrm{M}_{\odot}$ year $^{-1}$, whereas for the entire sample the mean is $\sim 200 M_{\odot}$ year $^{-1}$, consistent with the progenitors of these galaxies being starburst galaxies at $z \sim 5$ if the star formation persists at this rate for $\sim 1$ Gyr. Webb et al. (2006), in a separate study, find an average SFR of $130 M_{\odot}$ year $^{-1}$ for a similarly selected sample of 79 DRGs, in reasonable agreement with Papovich et al. As illustrated in Figure 2, Papovich et al. find that the SSFR for the DRGs at $1.5<z<3$ is about an order of magnitude larger than the SSFR for galaxies of the same mass in the redshift range of $0.3-0.7$. They conclude that DRGs, 


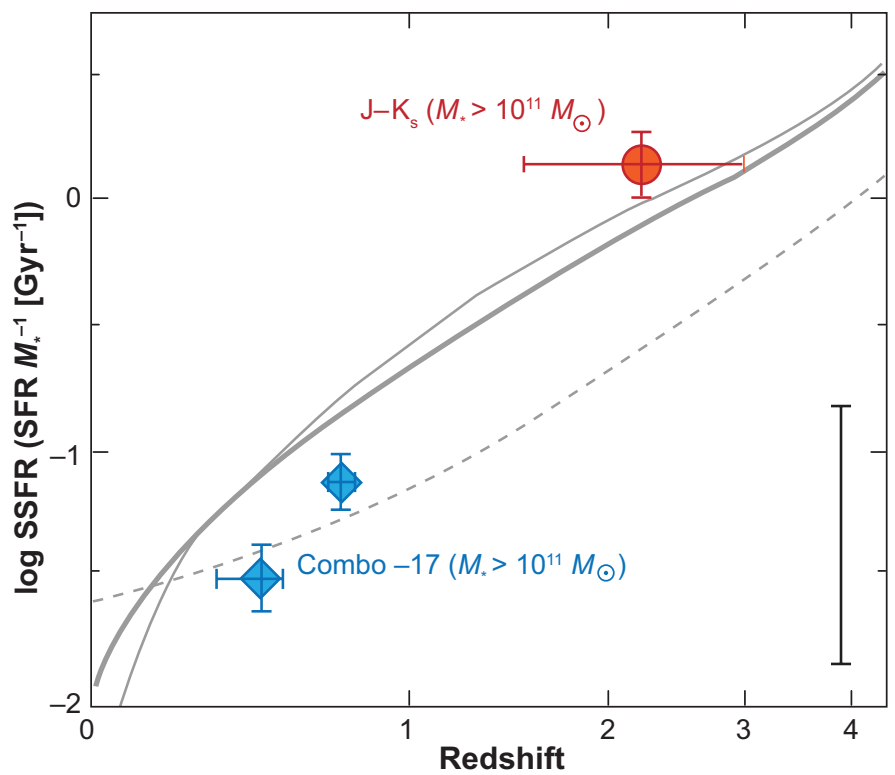

Figure 2

The evolution of the SSFR $\left(\dot{M} / M_{*}=S F R / M *\right)$ as a function of redshift $z$ (from Papovich et al. 2006, used by permission). The data points are from the work of Papovich et al. that utilize the Spitzer-derived SFRs, whereas the solid gray lines show the expected evolution of the ratio of the total SFR to the total galaxy stellar mass density (Cole et al. 2001, solid lines-the thick line includes correction for dust extinction), and the model of Hernquist \& Springel (2003, dashed line). The inset error bar represents the systematic uncertainties in the SFR.

representing massive galaxies at high redshift, contribute $10-30 \%$ of the total SFR in the range of $1.5<z<3$.

2.3.3. The thermal infrared emission from $z \sim 2$ galaxies. Daddi et al. (2005, 2007b) and Reddy et al. (2006) have explored thermal emission in star-forming galaxies at $z \sim 2$ using the deepest 24- $\mu \mathrm{m}$ Spitzer observations, the GOODS observations of the HDF-N and CDF-S fields. Daddi et al. focus on samples selected by the BzK algorithm [BzK selection (Daddi et al. 2004) is another color-based selection for identifying $z \sim 2$ galaxies and further subdivides these systems into star-forming and passively evolving systems], whereas Reddy et al. use samples selected based on LBG, BzK, and DRG criteria in the HDF-N field. Reddy et al. find that as a whole, the UVselected galaxies at $z \sim 2$ have $\left\langle L_{\mathrm{ir}}\right\rangle \sim 2.5 \times 10^{11} L_{\odot}$, which places them well into the LIRG range.

Daddi et al. (2007b) argue that $L_{\mathrm{ir}} / L_{\mathrm{uv}}$ values greater than 3 are due to obscured active galactic nuclei (AGN) or overestimating the bolometric corrections due to 8 - $\mu$ m (aromatic feature) emission stronger than seen in nearby galaxies of the same luminosity. Detailed studies of ULIRGs at $z \sim 2$ (see discussion in Section 4.2.3) have suggested that star-forming systems extend to $L>$ $10^{13} L_{\odot}$ at high redshifts, arguing that bolometric corrections $L_{\mathrm{ir}} / L_{\mathrm{uv}}>3$ are viable.

Based on the derived SFRs, Reddy et al. find the star-formation luminosity to be $\sim 75 \%$ IR (dustenshrouded) luminosity, and $\sim 25 \%$ UV luminosity with a large scatter in star-formation luminosity, ranging from $<10^{11} L_{\odot}$ to $>10^{12} L_{\odot}$, at a given galaxy mass independent of mass from $\sim 3 \times$ $10^{9} M_{\odot}$ to $\sim 3 \times 10^{11} M_{\odot}$, whereas Daddi et al. (2007b) find an SFR proportional to mass over the same range in masses. Whether these apparently differing results are due to differences in sample selection criteria or in analysis is unclear and highlights the early state of analyses of the Spitzer data. 
Daddi et al. $(2005,2007 \mathrm{~b})$ find that $\sim 40 \%$ of BzK galaxies with $M>10^{11} M_{\odot}$ are ULIRGs $\left(L_{\text {ir }}>10^{12} L_{\odot}\right)$, and that the lifetime of this state is $\sim 0.5$ Gyr. The inferred SFR and lifetime of the ULIRG phase at $z \sim 2$ lead to a near doubling of the stellar mass at that epoch. Caputi et al. (2006) find very similar results: that more than half of the most massive galaxies at $z \sim 2-3$ are ULIRGs, and therefore ULIRGs are a good tracer of the most massive galaxies at these redshifts.

Both Reddy et al. and Daddi et al. conclude that the buildup of mass in these galaxies must be nearly completed by $z \sim 1.5$. Caputi et al. (2007) find a total IR luminosity density at $z \sim 2$ of $\sim 6 \times 10^{8} L_{\odot} \mathrm{Mpc}^{-3}$, corresponding to an SFR of $0.1 \mathrm{M}_{\odot}$ year $^{-1} \mathrm{Mpc}^{-3}$. Reddy et al. (2008) suggest that the total IR energy density could be twice this. Assuming the SFR versus $z$ is constant between 3 and 1 (an interval of $3.6 \mathrm{Gyr}$ ) and integrating the SFR suggested by Caputi et al. lead to $\sim 4 \times 10^{8} M_{\odot} \mathrm{Mpc}^{-3}$ in formed stars (or $\sim 2 \times 10^{8} M_{\odot} \mathrm{Mpc}^{-3}$ locked into stars with a $50 \%$ recycling factor), consistent with the measured growth in stellar mass during that epoch.

2.3.4. Limits on the star-formation epoch in clusters of galaxies. Spitzer observations of clusters of galaxies have proven to be extremely useful at constraining the formation epoch of these systems. De Propris et al. (2007) have observed 32 clusters in the range $0.6<z<1.3$ and find that the majority of stellar mass in these clusters has formed by $z=1.3$. P.R.M. Eisenhardt, M. Brodwin, A.H. Gonzales, S.A. Stanford, et al. (submitted) detected 335 candidate clusters over the redshift range $0.1-1.9$, with $\sim 100$ having photometric redshifts greater than 1 . Ten of 11 spectroscopically observed clusters have been confirmed in the redshift range 1.06-1.41. Some of the spectroscopically confirmed $z>1$ clusters appear to have a red galaxy sequence in place. Comparing the $[\mathrm{I}]-[3.6 \mu \mathrm{m}]$ colors of the detected clusters with models of redshifted clusters suggests that up to $z \sim 1$, the cluster colors are well fitted by clusters forming at $z \sim 3$ in a 0.1 Gyr burst and then are evolving passively. For clusters with $z_{\text {phot }}>1$, the colors suggest higher formation epochs.

\subsection{The Farthest Reaches of the Universe $(z>4)$}

Spitzer has made substantial contributions to the study of galaxies beyond $z \sim 4$. The superb sensitivity of the IRAC short-wavelength channels has led to measurements of these highest redshift galaxies in the rest-frame visual. These observations are more sensitive to the contribution from more-evolved, lower-mass stars in the integrated galaxy light than are rest-frame UV observations.

Using IRAC images, Egami et al. (2005) detected a $z \sim 7$ galaxy, previously discovered as a lensed system behind the cluster Abell 2218 (Kneib et al. 2004). They determined a redshift $z$ in the range of 6.6 to 6.8 and suggested that the SED, shown in Figure 3, is consistent with a stellar population that possesses a fairly strong discontinuity in continuum flux between the rest UV and the rest visual as measured by the Spitzer observations. This is attributed to the Balmer break in the spectra of the stars in the lensed galaxy, suggesting an age that is surprisingly large (50$450 \mathrm{Myr}$ ) with a corresponding formation redshift as large as $z_{\text {form }} \sim 12$.

H. Yan et al. (2005a) utilized the extremely deep GOODS data to probe the rest-frame visible emission of galaxies identified as redshift 5 and 6 systems in the HUDF (Yan \& Windhorst 2004), detecting the brightest of the ACS-selected sample in the IRAC images. The vast majority of the detected galaxies were seen at $3.6 \mu \mathrm{m}$, with some also seen at $4.5 \mu \mathrm{m}$ in the IRAC observations. None of the galaxies were detected beyond $4.5 \mu \mathrm{m}$. They found a significant increase in flux from the rest UV to the rest optical. This rise is naturally produced by systems with stellar populations of ages of several hundred million years and implies masses of up to $10^{10} M_{\odot}$. This argues that galaxies of mass $10^{10} M_{\odot}$, similar to the LBGs at $z \sim 3$, were in place $\sim 10^{9}$ years after the Big Bang. 


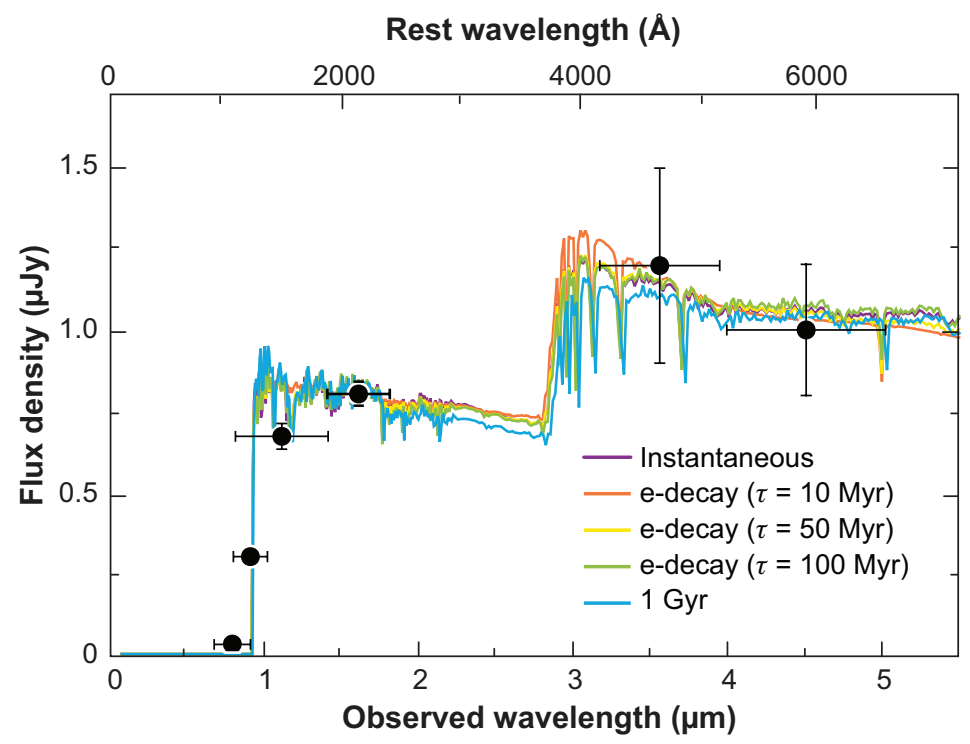

Figure 3

Model fits to the observed SED of the $z \sim 7$ galaxy reported by Egami et al. (2005, used by permission). The data at 3.6 and $4.5 \mu \mathrm{m}$ are from Spitzer, whereas the shorter wavelength data are from NICMOS and ACS on HST. The models represent an instantaneous burst, and exponentially decaying SFRs, and a constant SFR lasting 1 Gyr. The extinction to the forming stars is in the range $\tau_{\mathrm{v}}=0-0.5$. The best fit redshift $z$ is $\sim 6.6$.

H. Yan et al. were not able to place significant constraints on the metal abundances of these systems but found no need to invoke reddening. Some systems were found to be extremely blue in their rest-frame UV colors, demanding very young, massive stars with little reddening in these galaxies.

This work was followed up by more detailed studies of $z \sim 5$ systems by Stark et al. (2007) and $z \sim 6$ systems by Eyles et al. $(2005,2007)$ and H. Yan et al. (2006). Stark et al. utilize a sample of 14 spectroscopically confirmed galaxies in the redshift range of 4.4-5.6, and derive stellar masses and ages for these galaxies. By fitting SEDs to their larger sample of photometrically selected galaxies, they derive an estimate of the mass density in galaxies at $z \sim 5$ of $5-8 \times 10^{6} \mathrm{M}_{\odot} \mathrm{Mpc}^{-3}$, which increases to $>10^{7} M_{\odot} \mathrm{Mpc}^{-3}$ when accounting for non-star-forming galaxies at that redshift. Thus a few percent of the present stellar mass was in place as early as $z \sim 5$ (Figure 4).

H. Yan et al. (2006) and Eyles et al. $(2005,2007)$ focused on measuring galaxies at $z \sim 6$. The targets are selected from the GOODS fields based on nondetection in the $i$ band $(0.775 \mu \mathrm{m})$ with a minimum $(i-z))_{\mathrm{AB}}>1.3-1.5 \mathrm{mag}$. Eyles et al. (2005) studied four galaxies with spectroscopically confirmed redshifts of $\sim 5.8$, two of which were robustly detected at $3.6 \mu \mathrm{m}$. The $3.6-\mu \mathrm{m}$ detections immediately imply a $0.4-\mu \mathrm{m}$ break in the rest-frame, i.e., old galaxies. For these galaxies minimum (favored) ages are $100(250-650) \mathrm{Myr}$, and minimum (favored) masses are $10^{10}\left(1.3-3.8 \times 10^{10}\right)$ $M_{\odot}$. These galaxies suffer minimal reddening, and again the analysis is not sensitive to metallicity. $H$. Yan et al. expand their sample of $z \sim 6$ sources by considering sources not detected in the $\mathrm{V}$-band and with red $i-z$ colors. After filtering against foreground contaminants, they find a sample of 53 sources detected at $z$ and $3.6 \mu \mathrm{m}$, and 79 sources detected in $z$ but not detected in IRAC. Based on spectroscopic redshifts for three sources, they argue $z \sim 6$ is a reasonable estimate for all the sources in their sample.

Eyles et al. (2007), utilizing more extensive photometric and spectroscopic data in the CDF-S field, derive a minimum mass density of $2.5 \times 10^{6} \mathrm{M}_{\odot} \mathrm{Mpc}^{-3}$ at $z \sim 6$, consistent with that derived 


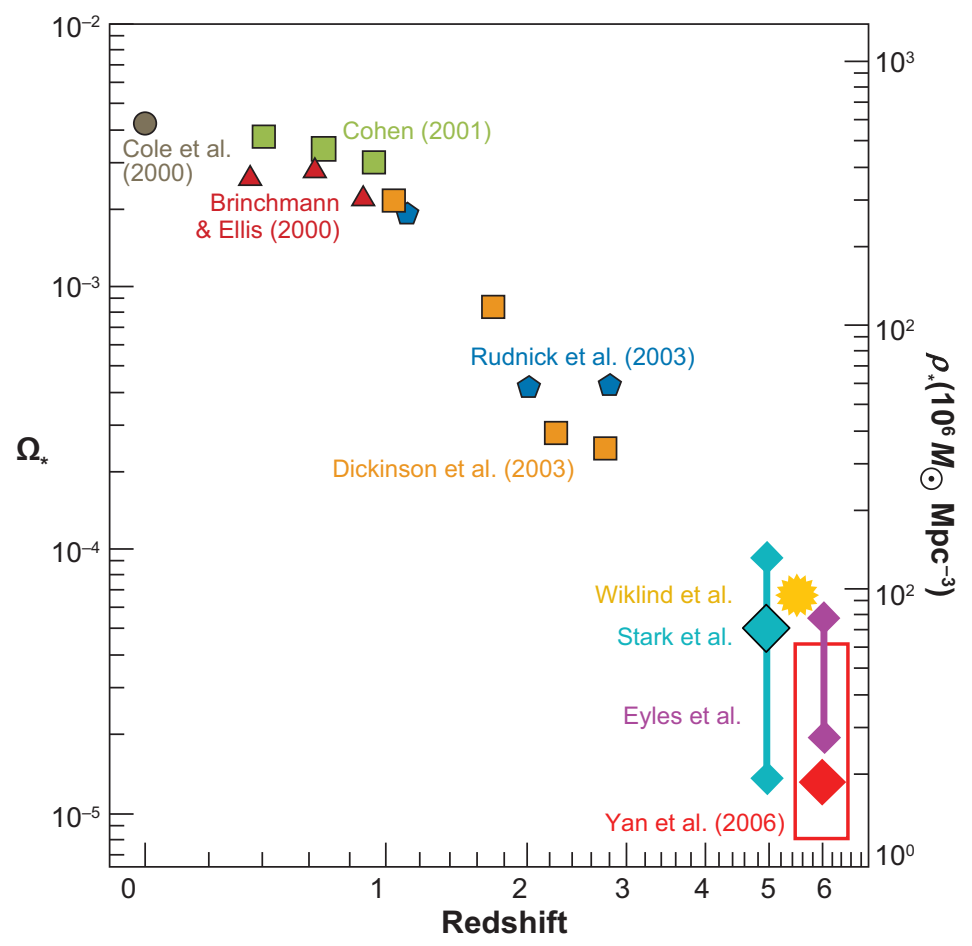

Figure 4

The mass density in stars as a function of redshift. The data for $z \sim 5$ and greater are from the Spitzer observations of H. Yan et al. (2006), Eyles et al. (2007), Stark et al. (2007), and Wiklind et al. (2008). Used by permission.

by H. Yan et al. of $1.6 \times 10^{6} M_{\odot} \mathrm{Mpc}^{-3}$ (Figure 4). Both H. Yan et al. and Eyles et al. suggest that the mass in galaxies at $z=6$ could be well above these lower limits, i.e., $\sim 5-8 \times 10^{6} \mathrm{M}_{\odot} \mathrm{Mpc}^{-3}$. Eyles et al. find that $6 / 9$ sources with IRAC detections have significant Balmer breaks, implying luminosity-weighted ages of 180-720 Myr at 0.9 Gyr after the Big Bang, and that $40 \%$ of the "i drop" sources contain "old" established stellar populations, whereas H. Yan et al. find, through image stacking, that the "IRAC invisible" population of $z \sim 6$ galaxies is significantly bluer and less massive than those detected with IRAC, with "representative" masses of $6 \times 10^{8} M_{\odot}$ and ages of 30 Myr.

Stark et al. (2007) point out that the observed mass at $z \sim 5$ is significantly greater than the SFR integrated over the redshift range of 5-10 (from, e.g., Bouwens et al. 2004, 2005, 2006), and suggest that this is a result of star formation either in dust-obscured regions at these redshifts (to which Spitzer would not be sensitive) or in lower-mass galaxies than have been detected to date. Eyles et al. (2007) point out that the increase in stellar mass density from $z=6$ to $z=5(\sim 5 \times$ $10^{6} M_{\odot} \mathrm{Mpc}^{-3}$ ) requires an SFR $\sim 4$ times that measured in this range by Bunker et al. (2004). From the range of star-formation histories of the galaxies that they find, they suggest that stars formed at $z>7$ could be responsible for a substantial fraction of the reionization of the universe.

Labbé et al. (2006) pushed the Spitzer detection of galaxies to $z \sim 7$ using J-band NICMOS detections with nondetections in the $z$ band. In the HUDF they detected two such sources at $3.6 \mu \mathrm{m}$ and one at $4.5 \mu \mathrm{m}$. The SEDs suggest photo-z's in the range of 6.8-7.4, with masses of 1-10 $\times 10^{9} M_{\odot}$ and SFRs of 3-12 $M_{\odot}$ year $^{-1}$. Mannucci et al. (2007) find no candidate $z \sim 7$ 
sources in the GOODS-South (CDF-S) field using shallower $\mathrm{J}$ and $\mathrm{K}_{\mathrm{s}}$ data. The two investigations appear to derive conflicting results, with Labbé et al. finding similar mass density in galaxies at $z \sim 7$ to that found at $z \sim 6$, whereas Mannucci et al. found an upper limit to the stellar mass $\sim 0.3$ of that at $z \sim 6$. Small number statistics and the substantially different sensitivity limits could have led to the differing conclusions.

Spitzer has characterized sources found via narrowband imaging of Ly $\alpha$ emission at $z=6.56$. Chary, Stern \& Eisenhardt (2005) found a peak at $4.5 \mu \mathrm{m}$ in the SED of a lensed Ly $\alpha$ source behind Abell 370, while Lai et al. (2007) detected three Ly $\alpha$ emitters in the GOODS-N (HDF) field at 3.6 and $4.5 \mu \mathrm{m}$. Chary et al. suggest that the peak in the SED at $4.5 \mu \mathrm{m}$ is a result of strong $\mathrm{H} \alpha$ emission from a young starburst galaxy of mass $\sim 10^{9} M_{\odot}$, with an SFR of $140 M_{\odot}$ year $^{-1}$ in a burst of age $5 \mathrm{Myr}$. Lai et al. find from the detections of the $\operatorname{Ly} \alpha$ emitters at $i, z, 3.6$, and $4.5 \mu \mathrm{m}$ that these galaxies have properties quite similar to those found via SED selections, i.e., masses $10^{9}-10^{10} M_{\odot}$ and ages 5-100 Myr.

Perhaps the most controversial detection of a galaxy at $z>5$ is that reported by Mobasher et al. (2005) of a J dropout source in the HUDF. The source (HUDF-JD2) is detected at H (NICMOS), $\mathrm{K}_{\mathrm{s}}$ (from the VLT), and all Spitzer bands from 3.6-24 $\mu \mathrm{m}$. The SED fitting suggests a post-starburst age of a few hundred million years at a redshift of $z \sim 6.5$ with a stellar mass of $6 \times 10^{11} M_{\odot}$. Such a large mass in an "old" galaxy at this redshift would present substantial challenges to theories of galaxy formation. Most surprising is the detection at $24 \mu \mathrm{m}$. Mobasher et al. suggest that this could indicate a hidden AGN. They identify an alternative but formally less probable model of a dusty galaxy at $z \sim 2.5$, in which case the $24-\mu \mathrm{m}$ emission would be a tracer of current star formation. Chary et al. (2007) and Dunlop, Cirasuolo \& McLure (2007) find evidence that the lower redshift $(z \sim 2)$ is more compelling.

As a follow-on to the work of Mobasher et al., Wiklind et al. (2008) report an extensive search for high-redshift and massive old galaxies in the GOODS-South area by searching for candidate systems with Balmer breaks traced by the criterion $\mathrm{K}_{\mathrm{s}}-[3.6]_{\mathrm{AB}}>1.2 \mathrm{mag}$. The choice of $\mathrm{K}_{\mathrm{s}}$ and $3.6 \mu \mathrm{m}$ for the Balmer-break wavelengths sets the redshift of the break to be $5<z<9$. The selection criteria identify galaxies with $z>5$ and age greater than 0.2 Gyr. They find 11 candidates (including JD-2 above), with an estimated completeness from their primary $\mathrm{K}_{\mathrm{s}}$ selection of $40 \%$. Seven of their 11 candidates have $24-\mu \mathrm{m}$ detections that might favor a lower redshift assignment. Wiklind et al. suggest these $24-\mu \mathrm{m}$ sources are dust-enshrouded quasars of bolometric luminosity $\sim 3 \times 10^{12} L_{\odot}$ associated with a host galaxy viewed at shorter wavelengths.

Wiklind et al. find the median $z$ of the 11 sources is 5.2 , and the average stellar mass is $2 \times$ $10^{11} M_{\odot}$. The ages of these systems range from $0.2-1 \mathrm{Gyr}$, and they have formation redshifts ranging from $z \sim 6$ to $z>25$. From the sample size, search volume, and estimated completeness, they estimate that such galaxies at $z>5$ represent more than $2-3 \%$ of the mass density of galaxies at $z=0$, a result that is apparently consistent with the work of Stark et al. (2007), Eyles et al. (2005, 2007), and H. Yan et al. (2006) (Figure 4). If the sources with 24- $\mu \mathrm{m}$ detections are not truly at this high redshift, then the estimate of mass density presented by Wilkind et al. drops by a factor of $\sim 5$.

Dunlop, Cirasuolo \& McLure (2007) and Rodighiero et al. (2007) have also carefully searched in the GOODS-South field for massive galaxies at $z>4$. Dunlop et al. searched for massive systems among galaxies brighter than $\mathrm{K}_{\mathrm{s}}=23.5$ and found no evidence for $M>3 \times 10^{11} M_{\odot}$ galaxies at $z>4$. Searching among fainter systems, Rodighiero et al. suggest $\sim 5$ objects might be massive galaxies at $z>4$.

Because of the large number of model parameters in the fits, it is not surprising that some very extreme sources found in the deepest Spitzer surveys can be interpreted as distant, massive galaxies. The quantitative determination of age and mass of these systems should be viewed as tentative until spectra are obtained [most likely with the fames Webb Space Telescope (JWST)]. 


\subsection{Number Counts, Background, and Angular Fluctuations at 3.6 and $4.5 \mu \mathrm{m}$}

Classical cosmology has utilized number counts and the measurement of backgrounds as powerful probes of the contents of the universe. Fazio et al. (2004) presented counts at 3.6 and $4.5 \mu \mathrm{m}$ to depths of 2.8 (20) and 1.8 (20) $\mu \mathrm{Jy}$ (Vega mag). These counts were limited by source confusion in the deepest integrations ( $\sim 8$ hours/sky pixel). Sullivan et al. (2007) extend the number counts at $3.6 \mu \mathrm{m}$ to $100 \mathrm{nJy}(23.5 \mathrm{mag})$, using the GOODS observations and applying careful completeness corrections to the measurements at the faintest flux levels. By integrating the flux from galaxies at $3.6 \mu \mathrm{m}$ to $40 \mathrm{nJy}$, Sullivan et al. determine a background due to the integrated light from galaxies of $6.6 \mathrm{nW} \mathrm{m}^{-2} \mathrm{str}^{-1}$. This should be compared to the Cosmic Background Explorer/Diffuse Infrared Background Experiment (COBE/DIRBE) measurement of $12.4 \pm 3.2 \mathrm{nW} \mathrm{m}^{-2} \mathrm{str}^{-1}$ (Wright \& Reese 2000), which is suggestive of a potentially significant discrepancy.

In highly controversial work, Kashlinsky et al. (2005, 2007a,b) analyzed a variety of deep IRAC observations and have found, after masking out the effects of individually detected sources, power at angular scales of $0.5^{\prime}-10^{\prime}$ in substantial excess above that attributed to foregrounds (at 3.6$5.8 \mu \mathrm{m}$ ) including zodiacal light, galactic emission, and galaxies at low redshifts. They attribute a lower limit of $1 \mathrm{nW} \mathrm{m} \mathrm{m}^{-2} \mathrm{str}^{-1}$ of the background power to Population III stars at $z>8$. Sullivan et al. (2007) and Cooray et al. (2007) have done independent clustering analyses on the same data, i.e., the deepest Spitzer images by GOODS with independent processing of the raw images. Cooray et al. place an upper limit of $0.4 \mathrm{nW} \mathrm{m}^{-2} \mathrm{str}^{-1}$ on a contribution of $z>8$ objects to the power detected on these angular scales, but suggest that a more plausible explanation of excess power is fluctuations due to unresolved fainter sources at $z<5$. Cooray et al. also place an upper limit of $7 \mathrm{nW} \mathrm{m}^{-2} \mathrm{str}^{-1}$ to the IR background at $3.6 \mu \mathrm{m}$ due to known galaxy populations. Whether this is significantly discrepant from the COBE/DIRBE-measured background is of great importance and is clearly critical to ascertaining whether Population III sources have been detected. It is likely that a definitive test will await the launch of JWST.

\subsection{Summary}

Deep Spitzer observations have enabled the direct measure of the dust-enshrouded star formation in galaxies to $z \sim 3$. The 24- $\mu$ m measurements of galaxies gauge their IR luminosity, and hence obscured star formation, which represents $\sim 75 \%$ of the total star formation in the universe. The results show a dependence of star-formation rate with redshift that is well matched to the opticalUV-based Lilly-Madau diagrams, with a peak in SFR at $z \sim 1$, an approximately constant rate to $z \sim 2.5$, and possibly a decrease in the SFR per volume at higher redshift. LIRGS (galaxies with $10^{11}<L_{\text {ir }}<10^{12} L_{\odot}$ ) dominate the comoving star-formation rate density for $z<1.5$. ULIRGs at $z \sim 2$ represent $\sim 40 \%$ of the massive $\left(>10^{11} M_{\odot}\right)$ galaxies at that epoch and are undergoing their final mass accumulation. The specific star-formation rate for massive galaxies $(\dot{M} / M *)$ increased by more than an order of magnitude from $z \sim 0.8$ to $z \sim 2$.

Many $z \sim 5-6$ galaxies in GOODS, the Hubble Ultra-Deep Field, and other deep surveys were detected at 3.6 and $4.5 \mu \mathrm{m}$ (rest-frame $0.5-0.7 \mu \mathrm{m}$ ). Their very red $\mathrm{K}-[3.6]$ and rather flat [3.6] - [4.5] colors reveal a strong spectral break, attributed to the Balmer continuum break in stellar populations dominated by stars with spectral classes from early B to mid-F. Ages for these galaxies derived from spectral synthesis models range from 100 to $500 \mathrm{Myr}$, corresponding to formation redshifts as large as $z_{\text {form }} \sim 12$, with inferred stellar masses as large as a few $\times 10^{10} M_{\odot}$ and perhaps higher. Based on the galaxies detected at these redshifts, $\sim 2-3 \%$ of the mass in stars was in place by $z \sim 5$. These stars are not adequate to reionize the universe, but lower mass or dust-enshrouded galaxies, undetectable even with the deepest Spitzer observations, remain a possible source of the reionization of the universe. Controversial but tantalizing results present 
candidate massive galaxies $\left(M>10^{11} M_{\odot}\right)$, at $z>5$ and evidence for population III stars from power spectrum analysis of the deepest IRAC data.

\section{THE FAR-INFRARED $(\lambda>20 \mu \mathrm{m})$ UNIVERSE}

\subsection{Number Counts in the Far-Infrared}

Number counts are an important tool for characterizing cosmic evolution and for testing detailed models of source populations. Indeed, the increased star-formation density between 0 and 1 described above should be reflected in number counts as well as in studies of particular source populations. To these ends, source counts to faint flux levels at high galactic latitude have been reported at 24, 70, and $160 \mu \mathrm{m}$. At the bright end of the Spitzer range, the data are consistent with previous counts from IRAS and ISO (Elbaz 2005). Like ISO and consistent with the increased star formation with redshift described in Section 2, Spitzer shows faint counts far in excess of the predictions of noevolution extrapolations. At all FIR wavelengths, particularly at $24 \mu \mathrm{m}$, where counts reach more than 3 orders of magnitude deeper than previous missions, and $70 \mu \mathrm{m}$, where the counts go much deeper than ISO, Spitzer's extensive surveys provide much larger samples than previously available.

3.1.1. $24 \mu \mathrm{m}$. The counts presented by Chary et al. (2004), Papovich et al. (2004), Marleau et al. (2004), and Rodighiero et al. (2006) show very similar results; the differential counts peak at $\sim 0.2-0.3 \mathrm{mJy}$, where the counts are more than an order of magnitude greater than a Euclidean extrapolation from the IRAS data at $100 \mathrm{mJy}$. The integral counts show 3000 sources per square degree that are brighter than $0.3 \mathrm{mJy}$. Below $0.3 \mathrm{mJy}$, the counts converge rapidly at a sub-Euclidian rate to the faint limit of the surveys, which is below $50 \mu \mathrm{Jy}$ in each case.

Lagache et al. (2004) show that the Spitzer MIPS counts as well as other data-including measurements of the FIR background-are adequately fit by a modification of an empirical model first applied to data from ISO and the Submillimeter Common-User Bolometer Array (SCUBA) (Lagache, Dole \& Puget 2003). This model incorporates templates based on both normal and starburst galaxies and extrapolates the local luminosity function with $z$, adjusting several parameters to fit the existing data on number counts and the IR background. They argue that the aromatic features at rest wavelengths $5-15 \mu \mathrm{m}$ remain strong at $z>2$, which is consistent with Spitzer spectroscopic studies tracing the aromatic emission to $z \sim 3$. Lagache et al. suggest that at $0.2 \mathrm{mJy}$, about $30 \%$ of the $24-\mu \mathrm{m}$ sources are at $z>2$ and that the IR energy output at $0.5<z<2.5$ is dominated by sources with $L_{\text {ir }}$ between $3 \times 10^{11}$ and $3 \times 10^{12} L_{\odot}$. Chary et al. (2004) reach essentially identical conclusions from analysis of the deep 24- $\mu \mathrm{m}$ counts in ELAIS-N1 and find that all but (at most) a few percent of the $24-\mu \mathrm{m}$ sources down to a limiting flux of $50 \mu \mathrm{Jy}$ have counterparts at 3.6 and/or $4.5 \mu \mathrm{m}$ to a detection limit below $1 \mu \mathrm{Jy}$. Gruppioni et al. (2005) have adopted an approach similar to that of Lagache et al. and extrapolated the ISOCAM 15- $\mu \mathrm{m}$ deep counts in ELAIS-N1 to predict, phenomenologically, the MIPS $24-\mu \mathrm{m}$ counts. Their results (Figure 5) show that the $15-\mu \mathrm{m}$ counts are good predictors of $24 \mu \mathrm{m}$ to fluxes below $100 \mu \mathrm{Jy}$. The models of Lagache et al. (2004) and Gruppioni et al. (2005) (among others) provide good fits to the number count measurements, but predict different distributions of redshifts in fluxlimited samples. Distinguishing these models requires large-scale redshift surveys that are only now becoming available. Desai et al. (2008b) have determined the distribution of redshifts of a complete sample of more than 500 sources that are brighter than $0.3 \mathrm{mJy}$ at $24 \mu \mathrm{m}$. They find qualitative agreement with the models of Lagache et al. but less so with those of Gruppioni et al. who predict a stronger peak at $z \sim 0.8$ than is observed. At lower flux levels, the models by Wang (2006) appear to provide the best fit to the observed redshift distribution. 


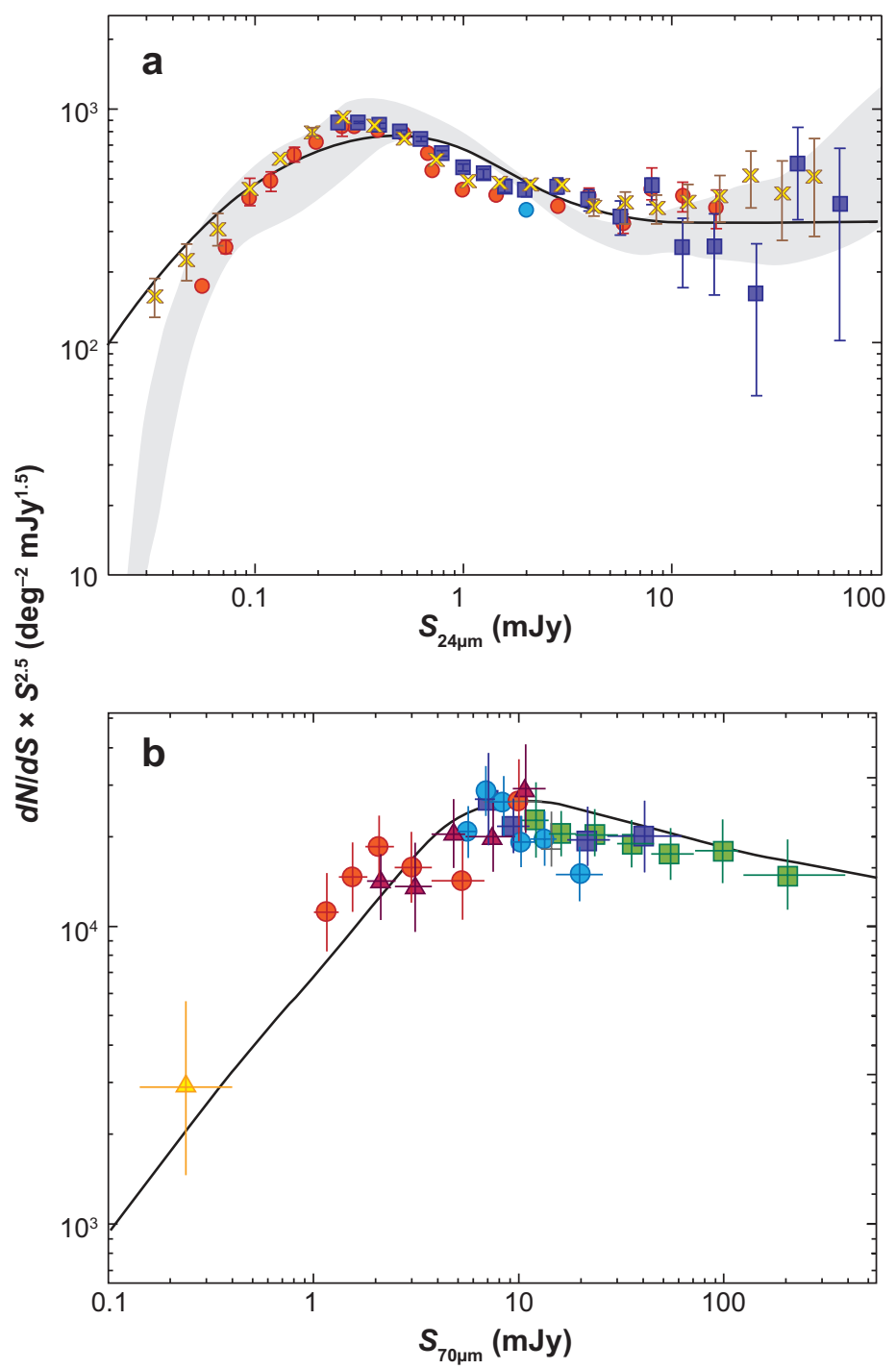

Figure 5

Galaxy counts at 24 and $70 \mu \mathrm{m}$, presented as excess over a Euclidean model versus source flux density. Consult the original papers (Gruppioni et al. 2005 at $24 \mu \mathrm{m}$, Frayer et al. 2006 at $70 \mu \mathrm{m}$ ) for information about the data sets presented (figures used by permission). (a) The gray area in the $24-\mu \mathrm{m}$ panel shows the range of predictions for $24-\mu \mathrm{m}$ counts as extrapolated from the ISOCAM $15-\mu \mathrm{m}$ sources, as described by Gruppioni et al. It falls off below $\sim 0.1 \mathrm{mJy}$ as the limiting flux density of the ISOCAM surveys is reached. (b) The $70-\mu \mathrm{m}$ counts at $0.3 \mathrm{mJy}$, which is the $1-\sigma$ confusion level, were derived by Frayer et al. using simulations to match the observed fluctuations.

3.1.2. $70 \mu \mathrm{m}$. Frayer et al. (2006) present the results of an ultradeep 70- $\mu \mathrm{m}$ survey in the GOODS-N field, reaching a $1-\sigma$ noise level of $\sim 0.3 \mathrm{mJy}$. They show that the differential source counts turn over at 8-10 mJy; below this flux level, the counts fall steeply with a slope comparable to that reported at $24 \mu \mathrm{m}$. Down to their limiting flux of $\sim 1.2 \mathrm{mJy}$, their number counts are fit tolerably well by the Lagache et al. (2004) models described earlier. At the bright end, the Frayer et al. results are consistent with those of Dole et al. (2004), who report a number density of 
240 galaxies/square degree brighter than $\sim 15 \mathrm{mJy}$, which exceeds the Euclidean extrapolation by a factor of $\sim 10$. The Frayer et al. counts are also shown in Figure 5; because the available 24- $\mu \mathrm{m}$ data are $\sim 100$ times more sensitive in flux density than even the ultradeep 70- $\mu \mathrm{m}$ data, there are (at most) only a few galaxies detected at $70 \mu \mathrm{m}$ but not at $24 \mu \mathrm{m}$.

Huynh et al. (2007a) extracted 143 sources brighter than $2 \mathrm{mJy}$ at $70 \mu \mathrm{m}$ from the GOODS-N data, almost all of which have $24-\mu \mathrm{m}$ counterparts and spectroscopic (116) or photometric (26) redshifts. They showed that the IR luminosity function varies substantially out to $z \sim 1$. Assuming a double power-law fit to the luminosity functions, the luminosity at the break increases by about an order of magnitude (from $10^{10.5}$ to $10^{11.5} L_{\odot}$ ) from redshift 0 to 1 . The $z \sim 1$ luminosity function extends almost to $10^{12} L_{\odot}$, where the density of galaxies is almost two orders of magnitude higher than locally. The evolution inferred from these data is similar to that found from 24- $\mu \mathrm{m}$ surveys by Pérez-González et al. (2005), Le Floc'h et al. (2005), and Caputi et al. (2007).

3.1.3. $160 \mu \mathrm{m}$. At $160 \mu \mathrm{m}$, the counts presented by Dole et al. (2004) extend down to $50 \mathrm{mJy}$, where $\mathrm{N}(>50 \mathrm{mJy}) \sim 290$ galaxies/square degree and the differential counts exceed the Euclidean extrapolation by $\sim 5$ times. Above $\sim 200 \mathrm{mJy}$, the counts agree with previous results from the ISO Far-Infrared Background (FIRBACK) survey at $170 \mu \mathrm{m}$. Dole et al. conclude that most of the sources responsible both for the $160-\mu \mathrm{m}$ counts and for the $70-\mu \mathrm{m}$ counts at $\sim 15 \mathrm{mJy}$ lie at $z \sim$ 0.7 , with a tail to $z \sim 2$.

\subsection{Cosmic Infrared Background Radiation}

The cosmological IR background (CIRB), due to the cumulative radiation from early galaxies, provides a key constraint on the history of star formation and accretion in the universe. The resolution of this IR background into individual sources has been a central scientific goal for Spitzer. The cosmic background contributed from sources resolved by Spitzer can be compared with estimates of the IR background from COBE to establish the fraction of the IR background resolved by Spitzer.

3.2.1. Measurements of the cosmic infrared background radiation. Based on COBE/DIRBE data, reviewed by Hauser \& Dwek (2001), there exists an isotropic IR background, peaking around $150 \mu \mathrm{m}$ with an intensity of $\nu I_{v}$ at the peak of $\sim 25 \mathrm{nW} \mathrm{m}^{-2} \mathrm{str}^{-1}$. Pre-Spitzer models of this background are presented in Lagache, Puget \& Dole (2005).

By integrating the counts in each Spitzer band it is possible to derive a lower limit to the background; this limit can be increased by extrapolating the counts to zero flux. In no case does this estimate disagree with direct measurements or upper limits on the background (Hauser \& Dwek 2001). The deep 70- $\mu \mathrm{m}$ counts of Frayer et al. (2006) account directly for most or all of the $70-\mu \mathrm{m}$ background. However, at $160 \mu \mathrm{m}$ the point sources detected by Spitzer fall far short of completely resolving the CIRB.

Dole et al. (2006) addressed the shortfalls by exploiting the smaller beam and substantially higher sensitivity at $24 \mu \mathrm{m}$ (compared to the longer wavelengths) to enhance the depth at the longer wavelengths via stacking analysis. Using the stacking technique, Dole et al. (2006) determine the contribution of the $24-\mu \mathrm{m}$ detected sources to the CIRB at 70 and $160 \mu \mathrm{m}$. The average 100$\mu \mathrm{Jy} 24-\mu \mathrm{m}$ source has 70 - and $160-\mu \mathrm{m}$ flux densities of $\sim 0.5$ and $\sim 3 \mathrm{mJy}$, respectively, which is fainter than the estimated confusion limit at these wavelengths. Their results are included in Table 1, which gives the contribution to the CIRB at 24, 70, and $160 \mu \mathrm{m}$ from galaxies individually detected at $24 \mu \mathrm{m}$ and from fainter $24-\mu \mathrm{m}$ sources based on an extrapolation of the Spitzer data. Also included in Table 1 is the Dole et al. best estimate of the total CIRB. At both 70 and $160 \mu \mathrm{m}$, 
Table 1 Contribution of 24- $\mu \mathrm{m}$ galaxies to the CIRB (Dole et al. 2006)

\begin{tabular}{l|c|c|c}
\hline Wavelength & $24 \mu \mathrm{m}$ & $70 \mu \mathrm{m}$ & $160 \mu \mathrm{m}$ \\
\hline Background due to sources $>60 \mu \mathrm{Jy}$ & $2.16 \pm 0.26$ & $5.9 \pm 0.9$ & $10.7 \pm 1.6$ \\
\hline Contribution from sources $<60 \mu \mathrm{Jy}$ & 0.54 & 1.2 & 2.6 \\
\hline Total from $24-\mu \mathrm{m}$ sources & 2.7 & $7.1 \pm 1.0$ & $13.5 \pm 1.7$ \\
\hline Best estimate of total background & 2.7 & 6.4 & 15.4 \\
\hline
\end{tabular}

Note: All brightnesses are in units of $\mathrm{nW} \mathrm{m}^{-2} \mathrm{str}^{-1}$.

the results show that galaxies detected by MIPS at $24 \mu \mathrm{m}$ account for essentially all of the CIRB (Figure 6). Dole et al. attribute the $24-160-\mu \mathrm{m}$ CIRB to galaxies typically at $z \sim 1$, with $\sim 1 / 4$ of the background from galaxies at $z>1.5$. These galaxies are LIRGs with bolometric luminosities $\sim 10^{11}$ to $10^{12} L_{\odot}$, SFRs of $\sim 20-130 M_{\odot}$ /year, and masses $10^{10}-10^{11} M_{\odot}$ (see Sections 2.2. and 2.3). Dole et al. show that the CIRB (wavelengths $8-800 \mu \mathrm{m}$ ) has a total brightness of $24 \mathrm{nW} \mathrm{m}^{-2} \mathrm{str}^{-1}$, essentially equal to that of the UV/optical/near-IR background $(0.1-8 \mu \mathrm{m})$, which they estimate to be $23 \mathrm{nW} \mathrm{m} \mathrm{mstr}^{-1}$. Thus, half of the energy generated by nuclear burning or gravitational accretion over cosmic time has been absorbed by dust and reradiated in the IR.

Lagache et al. (2007) report detection of correlated variations in the $160-\mu \mathrm{m}$ background radiation over spatial scales from $5^{\prime}$ to $30^{\prime}$. These appear to be in excess of the expected contribution from cirrus and are attributed to clustering of IR galaxies at the redshift of the galaxies producing the background. Other data sets, such as the other SWIRE fields (Lonsdale et al. 2003) or the recently completed S-COSMOS Spitzer survey (Sanders et al. 2007), should be analyzed to verify this result.

3.2.2. Correlations of cosmic infrared background radiation with other phenomena. Dye et al. (2007) have coadded 70 - and $160-\mu \mathrm{m}$ images at the positions of the $23(850-\mu \mathrm{m})$ SCUBA sources brighter than $3.5 \mathrm{mJy}$ detected in the Canada-UK Deep Submillimetre Survey (CUDSS) deep survey field. Assuming the CIRB values given for these wavelengths by Dole et al. (2006), they can attribute only $\sim 15 \%$ of the background at either 70 or $160 \mu \mathrm{m}$ to the SCUBA sources. In agreement with the results of Dole et al., Dye et al. conclude that the CIRB is not due to a rare population of extremely luminous submillimeter sources.

\subsection{Active Galactic Nuclei}

The relative amount of energy generated by nuclear burning and by gravitational accretion as a function of environment and cosmic time is a fundamental parameter characterizing the evolution of the universe. Spitzer can contribute to this assessment because star formation and accretion (AGN) powered luminosities present different signatures in the IR. Spitzer searches for dustenshrouded AGN establish AGN contributions to the luminosities of IR-bright galaxies and to the IR background, assess the relative roles of star formation and AGNs in powering IR sources as a function of redshift, and perhaps even monitor the simultaneous buildup of stellar mass and black hole mass with cosmic time.

3.3.1. Spitzer observations of known luminous $\mathrm{X}$-ray sources. The minimum contribution of AGN to the IR energy budget is estimated through observations of known luminous X-ray sources, as X-ray sources with $L_{\mathrm{x}}>1 \times 10^{43} \mathrm{erg} \mathrm{s}^{-1}$ are considered to be AGN (Barger et al. 2005). Brand et al. (2006) have studied $\sim 14,00024-\mu \mathrm{m}$ detected galaxies in the NDWFS Boötes field at $z>$ 0.6 that have visible counterparts and, in almost all cases, IRAC $8-\mu \mathrm{m}$ detections. Histograms of 
Frequency $v(\mathrm{GHz})$

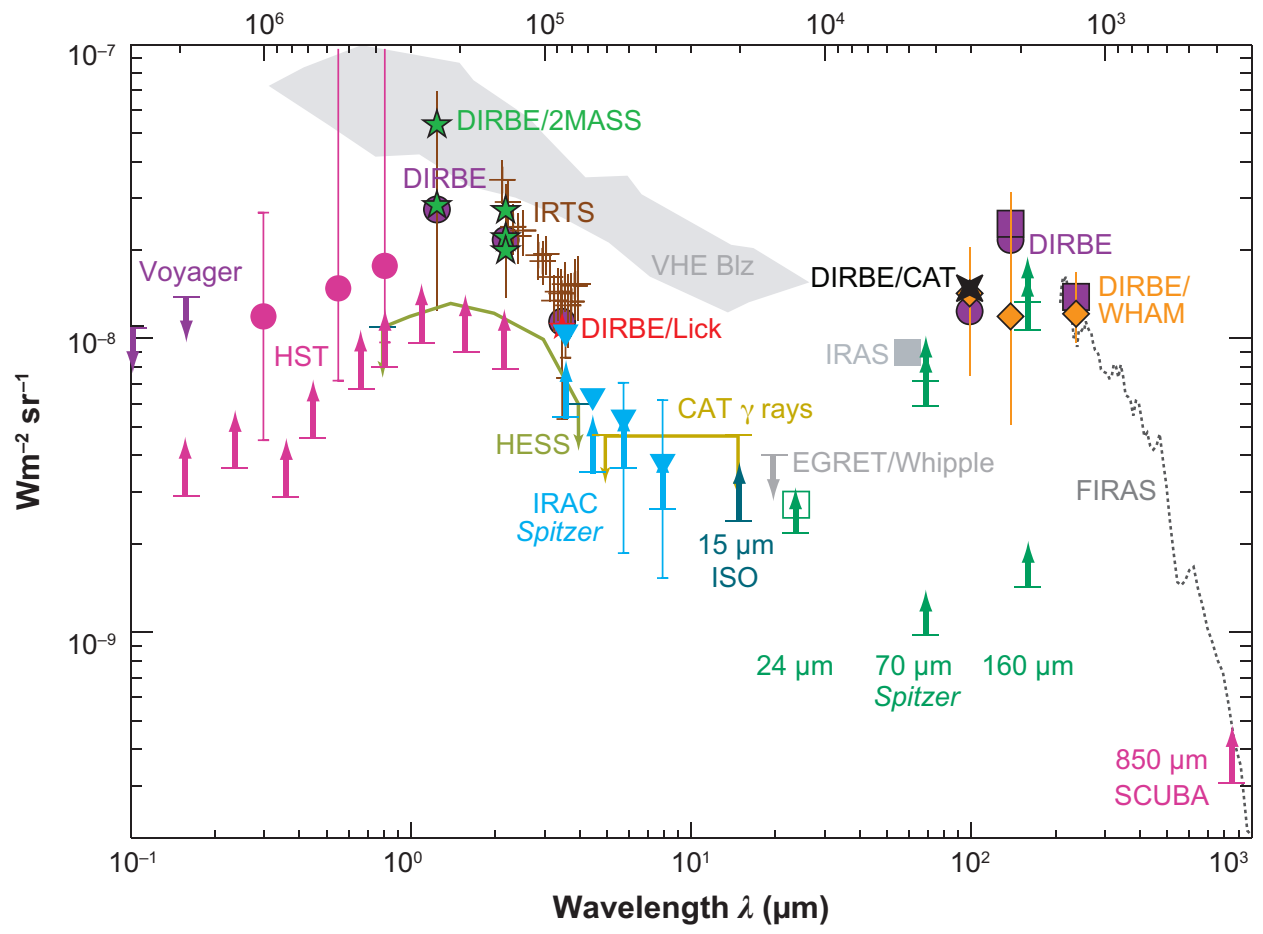

Figure 6

The extragalactic background light spectral energy distribution from 0.1 to $1000 \mu \mathrm{m}$, adapted from Dole et al. (2006, figure used by permission). At Spitzer wavelengths from 24-160 $\mu \mathrm{m}$, the green arrows are lower limits based on directly observed sources. The higher green arrows at 70 and $160 \mu \mathrm{m}$ are based on stacking numerous 24- $\mu \mathrm{m}$ detections, as discussed in Dole et al. whereas the highest green arrows at these wavelengths reflect the estimated contribution of all $24-\mu \mathrm{m}$ sources, including those beyond the sensitivity limit of the stacking analysis. The green open square is the estimated $24-\mu \mathrm{m}$ background when these faint sources are included. Note that the corrected $70-$ and $160-\mu \mathrm{m}$ background estimates from the MIPS data are essentially equal to the backgrounds estimated at these wavelengths by other techniques. See Dole et al. (2006) for identification of the other data sets and background estimates included in this figure.

$Q=\log \left\{v f_{v}(24 \mu \mathrm{m}) / \nu f_{v}(8 \mu \mathrm{m})\right\}$ show a pronounced peak at $Q \sim 0$, which they associate with AGN domination $\left(Q=0\right.$ corresponds to a flat spectrum in $v f_{v}$, typical for AGN). The identification of this peak as due to AGN is quite unambiguous because the subset (about 1000) of galaxies with $\mathrm{X}$-ray detection clusters tightly around $Q \sim 0$. The detection of these $z>0.6$ objects in the relatively shallow Chandra survey of the NDWFS guarantees that they have AGN-like X-ray luminosities. Similarly, a subset of $\sim 1000$ galaxies with AGN-like optical spectra cluster tightly at $Q \sim 0$. A secondary peak is seen at $Q \sim 0.5$, which is identified spectroscopically with highredshift starbursts; and galaxies with mixed AGN/starburst spectra lie at intermediate values of $Q$. Brand et al. find that the AGN fraction for $z>0.6$ galaxies increases from $\sim 10 \%$ for objects with $f_{v}(24 \mu \mathrm{m}) \sim 0.4 \mathrm{mJy}$ to $\sim 75 \%$ for those with $f_{v}(24 \mu \mathrm{m})>1.9 \mathrm{mJy}$. They estimate that AGNdominated sources contribute $3-7 \%$ to the $24-\mu \mathrm{m}$ background. This is a lower limit because some objects are deeply enough embedded that their observed $8-\mu \mathrm{m}$ emission is suppressed; in addition, it does not include the AGN contribution to the energy output of sources that are starburst-dominated, such as the ULIRGs discussed below. 
Treister et al. (2006) carried out a similar analysis using deeper observations, particularly in the $\mathrm{X}$-ray bands, but in a much smaller solid angle. Treister et al. estimate the AGN contribution to the $24-\mu \mathrm{m}$ background at $\sim 15 \%$, which is in acceptable agreement given the uncertainties in such estimates. They also estimate the contribution of AGN to the backgrounds in the IRAC bands, finding it to be increasing from $\sim 3.5 \%$ at $3.6 \mu \mathrm{m}$ to $\sim 8 \%$ at $8 \mu \mathrm{m}$. Treister et al. further report that AGN with $L_{\mathrm{x}}>1 \times 10^{42} \mathrm{erg} \mathrm{s}^{-1}$ have median IR luminosities at both 3.6 and $8 \mu \mathrm{m}$ that are 10-15 times greater than those of nonactive galaxies at the same redshift and that the $\mathrm{X}$-ray to IR luminosity ratio resembles that of AGN in the local universe. Ballantyne \& Papovich (2007) use detailed SED modeling at X-ray to IR wavelengths to estimate the AGN contribution to the IR background to be $\sim 10 \%$.

3.3.2. Identification of active galactic nuclei through continuum colors and spectral energy distributions. Papovich et al. (2007), studying the CDFS/GOODS-S field, show through stacking analysis that galaxies brighter than $250 \mu \mathrm{Jy}$ at $24 \mu \mathrm{m}$ have lower 70 - and $160-\mu \mathrm{m}$ fluxes, and hence lower bolometric luminosities, than implied by commonly used star-forming templates. Fainter galaxies in this redshift range are adequately described by these templates. They find that the $70-\mu \mathrm{m} / 24-\mu \mathrm{m}$ flux ratios of these (relatively) bright $24-\mu \mathrm{m}$ sources resemble those of sources with X-ray detections and suggest that the excess $24-\mu \mathrm{m}$ emission is due to AGN activity. This suggests that SFRs for $z \sim 2$ galaxies that are bright at $24 \mu \mathrm{m}$ can be overestimated by factors of $\sim 3-10$ if based entirely on fitting local templates to the observed $24-\mu \mathrm{m}$ data. Papovich et al. conclude, based on the full 24- to 160- $\mu \mathrm{m}$ SEDs, that nearly all $24-\mu \mathrm{m}-$ selected galaxies have $L<$ $6 \times 10^{12} L_{\odot}$ and SFRs less than $10^{3} M_{\odot}$ year $^{-1}$. Because even the Spitzer observations at $160 \mu \mathrm{m}$ correspond to $\sim 50 \mu \mathrm{m}$ in the rest-frame of these objects, this conclusion should be tested with longer wavelength observations afforded by the (soon to be launched) Herschel Space Observatory and submillimeter observations from the ground.

Daddi et al. (2007a) have obtained similar results for a sample of $24-\mu \mathrm{m}$ detected galaxies with $1.4<z<2.5$ in both GOODS fields, comparing the $24-\mu \mathrm{m}$ data with that in several other spectral bands from UV-visible to X-ray. After excluding sources detected by Chandra, they find that many of the brighter $24-\mu \mathrm{m}$ sources show excess $24-\mu \mathrm{m}$ emission over what would be expected from the SFRs implied by other indicators. These IR-excess sources occur most frequently in the more massive galaxies in the sample, as inferred from their $\mathrm{K}$ magnitudes. By stacking the Chandra $\mathrm{X}$-ray data at the positions of both the "normal" galaxies and those with a $24-\mu \mathrm{m}$ excess, they find that the former show a soft X-ray spectrum that is consistent with a star-forming galaxy. The latter, however, show a much harder spectrum; the spectrum and the intensity of the X-ray emission are evidence for highly obscured or even Compton-thick AGN. They argue that the reprocessed energy from these buried AGN is responsible for the excess 24- to 70- $\mu \mathrm{m}$ radiation. The space density of the Compton-thick AGN is $\sim 2.6 \times 10^{-4} \mathrm{Mpc}^{-3}$, higher than that of all AGN previously known at $z \sim 2$. These buried AGN make a significant but not dominant contribution to the "missing" X-ray background in the $10-30 \mathrm{keV}$ range. Finally, and most intriguingly, the presence of obscured AGN within massive, star-forming galaxies offers the first direct observation of the concurrent growth of the massive black hole and the stellar mass of the hosting galaxy. Daddi et al. suggest that these objects may approach the black hole mass/stellar bulge mass relationship. Lacy et al. (2007) (and others whose work is summarized therein) reinforce this conclusion and show that obscured objects dominate the AGN population over a wide range of luminosities and redshifts.

Lutz et al. (2005) find a similar result in their millimeter-wavelength study of $4024-\mu \mathrm{m}$ bright, optically faint extragalactic sources from the First Look Survey (FLS). As a group, these sources, though detectable, are considerably fainter than would be expected from the redshifted 
SEDs of local star-forming galaxies. This requires a strong, but optically obscured, mid-IR component without commensurate far-IR emission. They suggest that these sources may be luminous, optically obscured AGN that could represent a significant part of the high-redshift AGN population.

3.3.3. Submillimeter galaxies. Spitzer's ability to distinguish accretion-powered from stellarpowered sources has also been brought to bear on the study of the poorly understood submillimeter galaxy (SMG) population [SMGs are galaxies bright at submillimeter wavelengths that account for a good fraction of the cosmic background in this wavelength band (see Blain et al. 2002 for a review)]. Several groups have studied SMGs with Spitzer, both spectroscopically and photometrically. The spectroscopic results reported by Valiante et al. (2007) and Pope et al. (2008) are of particular interest. They obtained IRS spectra of 13 bright SMGs for which accurate positions are available. Nine of these were detected, and the spectra are heavily dominated by the aromatic-emission features at rest wavelengths between 5 and $10 \mu \mathrm{m}$. This implicates star formation as the main energy source for these SMGs, based on the known anticorrelation of aromatic feature strength and AGN activity (Section 4.2.1). The redshifts determined from the Spitzer data agreed with those previously available for six of these sources; for the other three, Spitzer provides the first redshift estimate. For this sample of objects, the median redshift is $2.7-2.8$, depending on how the nondetections are treated. An underlying mid-IR continuum that might be attributable to AGN activity is at most weakly present. Valiante et al. thus conclude that the SMGs are scaled-up versions of local ULIRGs, with median luminosity approaching $1 \times 10^{13} L_{\odot}$; these extremely prolific star-forming objects may represent a dramatic phase in the early evolution of galaxies. The average rest-frame $6-8.5 \mu \mathrm{m}$ spectrum of the nine detected SMGs closely resembles that of the HII-like ULIRG spectra reported by Desai et al. (2007) and does not differ greatly from the local starburst template provided by Brandl et al. (2006).

Pope et al. (2006) present multiband photometric data, including Spitzer $24-\mu \mathrm{m}$ measurements, for about three dozen SCUBA sources from the GOODS-N field. By comparison with local templates, they find a median IR luminosity of $6.0 \times 10^{12} L_{\odot}$ and a median redshift of 2.0. Consistent with the discussion above, they also find that AGN activity is a minor contributor to the energy output of SMGs. They also report that the SMGs appear to have lower temperature dust than do local ULIRGs of the same luminosity, but selection effects may be influencing this conclusion. Huynh et al. (2007b) have studied this sample of objects at $70 \mu \mathrm{m}$, reporting two detections and a stacking analysis, which shows that, on average, the lower redshift $(z<2)$ objects are brighter at $70 \mu \mathrm{m}$ than those at higher redshift. Confining themselves to these lower redshift objects, which have a median $z \sim 1.4$, they estimate an average IR luminosity of $8.0 \times 10^{11} L_{\odot}$ and a lower dust temperature than seen in local ULIRGs. They suggest that the SEDs are best fit by scaled-up versions of local spiral galaxy SEDs and estimate that the AGN contribution to the luminosity of the lower redshift SMGs is less than $25 \%$.

Younger et al. (2007) have combined Spitzer data with observations at other bands to study a population of SMGs with faint (or no) radio counterparts. Key to the success of this work was their ability to obtain accurate $\left(\sim 0.3^{\prime \prime}\right)$ positions of the SMGs using the Submillimeter Array. Six of the seven targets were detected by IRAC, but overall these objects are systematically fainter in the Spitzer bands than are radio-bright SMGs. Younger et al. suggest that these SMGs are very dusty starbursts at $z>3$ that have luminosities in the range of $1.0 \times 10^{13} L_{\odot}$, and are faint in the radio because of their high redshifts.

Dey et al. (2008) have speculated that an evolutionary connection exists between the 24- $\mu \mathrm{m}-$ selected and SMG populations whereby the SMGs evolve into the $24-\mu \mathrm{m}$ bright, high-luminosity 
sources. This would explain the correlation between black hole and stellar mass that is observed for galaxies in the local universe.

\subsection{Summary}

Spitzer's extensive wide and deep surveys reveal many more sources at low flux levels than expected from a Euclidean extrapolation of the local source population; this is qualitatively consistent with the increase in star-formation density out to $z \sim 1$ discussed earlier. At the same time, the discrete sources seen at $24 \mu \mathrm{m}$ can account for all of the CIRB radiation between $\sim 20$ and $\sim 200 \mu \mathrm{m}$, which can thus be attributed in large part to star-forming galaxies concentrated between $z \sim 1$ and $z \sim 1.5$.

The Spitzer results summarized above suggest that, at $z>2,24-\mu \mathrm{m}$ sources brighter than $0.3 \mathrm{mJy}$ are dominated by AGN activity, but also show that luminous AGN contribute at most $\sim 20 \%$ of the IR background. There is evidence as well for a substantial population of obscured, heavily embedded or Compton-thick AGN that contribute significantly to the black hole or hard X-ray budget of the universe. Conversely, at $z \sim 2$, the SMG population appears to be starburst-dominated. Perhaps we are witnessing in these two populations (at the same redshift) the simultaneous buildup of black hole mass and stellar mass.

\section{INFRARED EMISSION FROM GALAXIES: PROPERTIES AND PROCESSES}

\subsection{The Low-Intensity, Nearby Universe}

Spitzer observed in varying degrees of spatial and spectral detail a large number and variety of galaxies in the nearby universe drawn from the luminosity range of $\sim 10^{8}$ to $\sim 10^{11} L_{\odot}$, resolving thousands, mostly at the shortest wavelengths. It has imaged a few hundred nearby galaxies, gauging the distribution of stars at 3.6 and $4.5 \mu \mathrm{m}$, dust at 5.8 to $160 \mu \mathrm{m}$, and gas (in IRS line maps) at spatial resolution ranging from a few parsecs to $\sim 1 \mathrm{kpc}$.

4.1.1. Imaging studies. The Large Magellanic Cloud (LMC) (Meixner et al. 2006, Figure 7), Small Magellanic Cloud (SMC) (Bolatto et al. 2007), M31 (Barmby et al. 2006, Gordon et al. 2006, Figure 8), and M33 (Hinz et al. 2004) reveal the greatest detail, whereas the 75 galaxies of the Spitzer Infrared Nearby Galaxies Survey (SINGS; Kennicutt et al. 2003; Figure 9) cover a variety of galaxy types and luminosities. M51 data (Kennicutt et al. 2007) have provided a detailed laboratory for analyzing local SFRs within a galaxy (Figure 10).

Regardless of spatial resolution, galaxy images at 3.6 and $4.5 \mu \mathrm{m}$ reveal to first order a lowextinction view of the photospheric light. The longer-wavelength images are dominated by interstellar dust emission. Aromatic features contribute or dominate at 5.8 and $8 \mu \mathrm{m}$, stochastically heated very small grains are most important at $24 \mu \mathrm{m}$, and large grains dominate at 70 and $160 \mu \mathrm{m}$ (Désert, Boulanger \& Puget 1990, Dale et al. 2001). Photometry at 3.6 and $4.5 \mu \mathrm{m}$ is valuable in a number of applications, from extending the mass-metallicity relation to lower masses (Lee et al. 2006) to improving the stellar synthesis models of evolving galaxies (Maraston et al. 2006). The bands at 5.8 and $8 \mu \mathrm{m}$ contain emission from both stars and dust, and normal practice is to remove the stellar contribution estimated from 3.6 and $4.5 \mu \mathrm{m}$ to obtain "dust-only" maps (Helou et al. 2004, Pahre et al. 2004a, Smith et al. 2007). Hot dust, concentrated on HII regions, has been detected at $4.5 \mu \mathrm{m}$, both in spiral galaxies (Helou et al. 2004) and in irregulars (Hunter, Elmegree \& Martin 2006). Spitzer data also detect dust associated with mass-losing evolved stars in early-type galaxies (Bressan et al. 2006). 


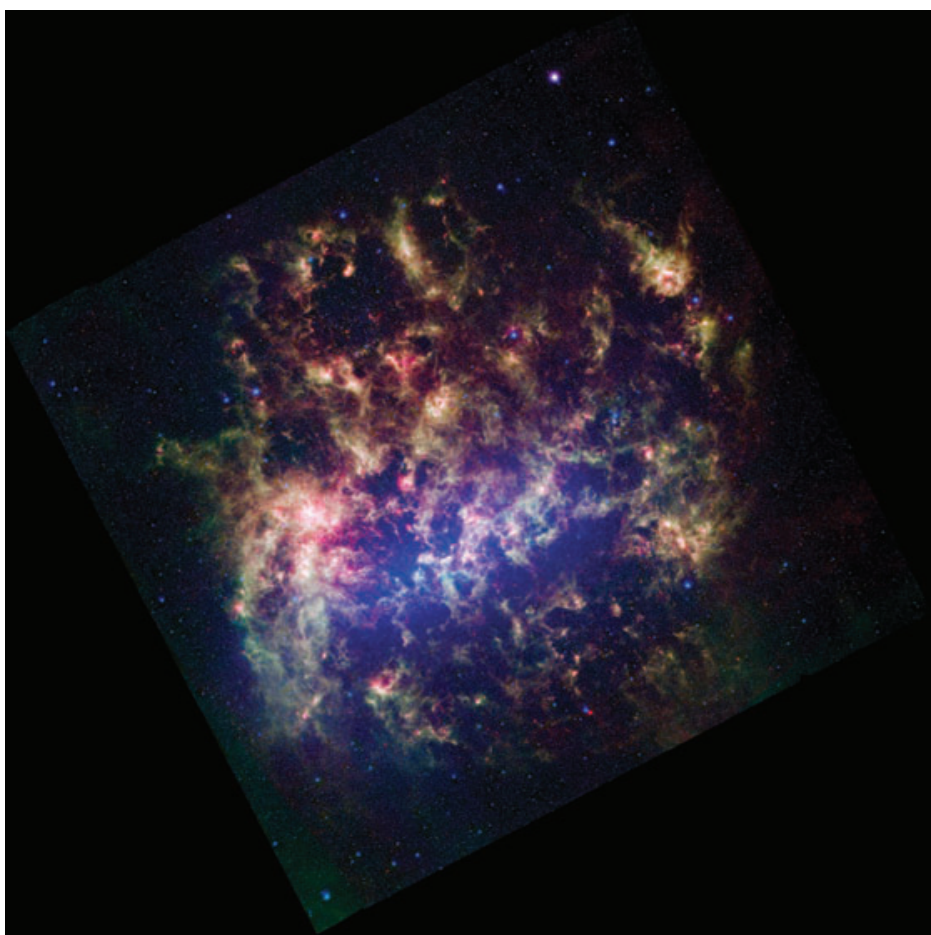

Figure 7

The Large Magellanic Cloud as imaged by Spitzer at 3.6 (blue), 8 (green), and 24 (red) $\mu \mathrm{m}$ (Meixner et al. 2006, figure used by permission). The stellar bar is evident as the diffuse blue structure across the middle of the image. Note the variation in the colors of dust emission, from areas with elevated $24 / 8-\mu \mathrm{m}$ color ratios seen as red, to areas with elevated $8 / 24-\mu \mathrm{m}$ color ratios manifested as green and associated with less intense emission away from peak star-formation activity.

Hogg et al. (2005) combined photometry from IRAC and the Sloan Digital Sky Survey (SDSS) for 300 galaxies and found that optically blue galaxies are richer in aromatic feature emission than red ones, as expected for galaxies active in star formation. Low-luminosity blue galaxies are, however, poorer in aromatic feature emission, pointing to distinct behavior among dwarf galaxies as detailed below. Dale et al. (2007), in a study of SINGS galaxies with Spitzer and Galaxy Evolution

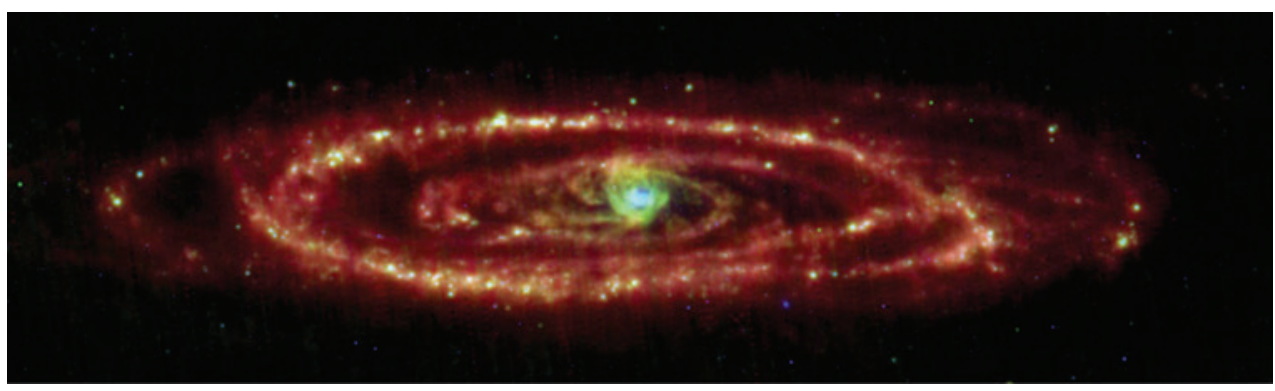

Figure 8

The Andromeda Nebula, Messier 31 as imaged by Spitzer at 24 (blue), 70 (green), and 160 (red) $\mu \mathrm{m}$. The excess of blue/green colors in the nuclear region signals warm dust in circumstellar shells in the bulge (Gordon et al. 2006, figure used by permission). 


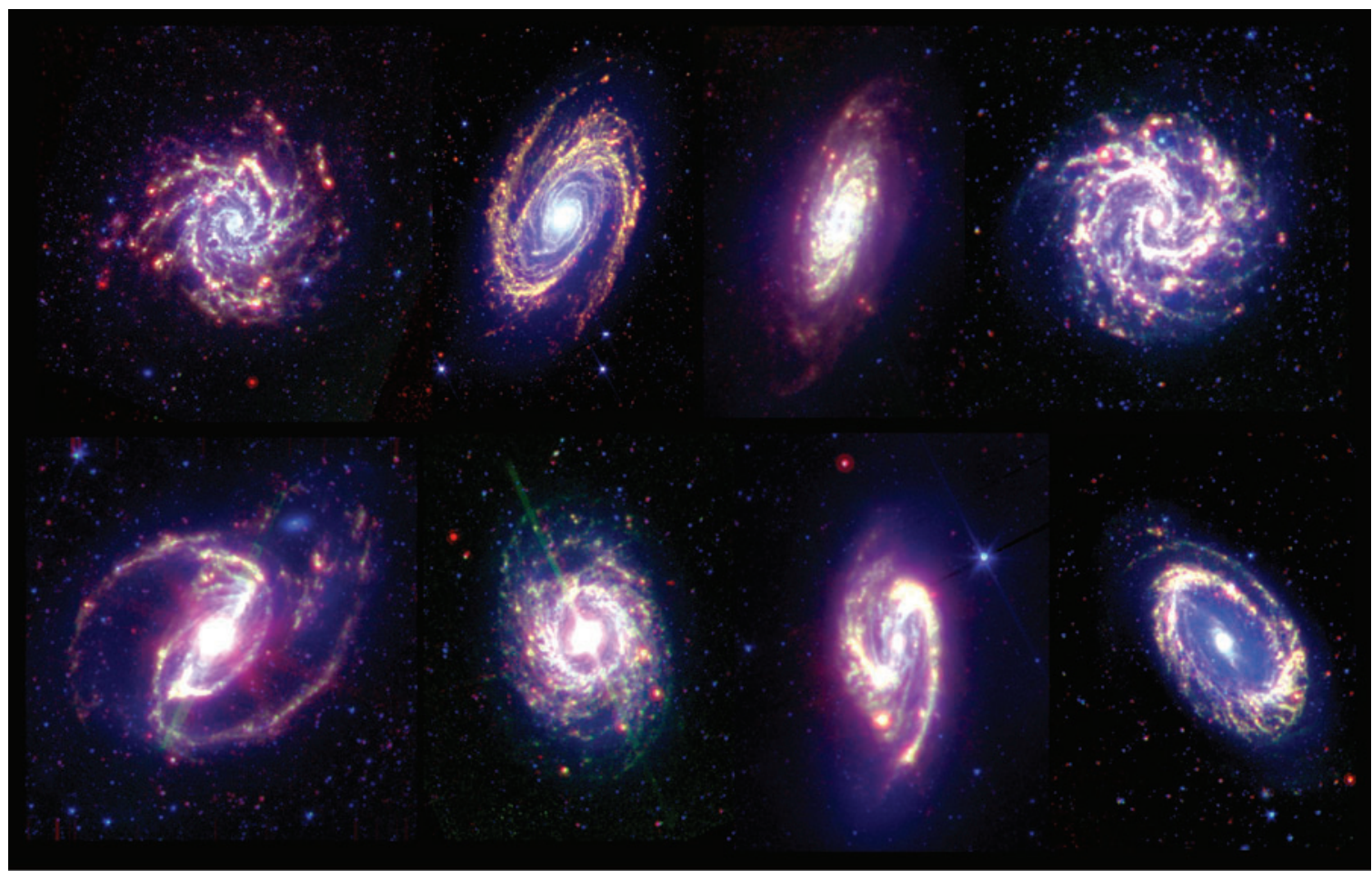

Figure 9

Selected SINGS spiral galaxies (Kennicutt et al. 2003) as imaged by Spitzer at 3.6 (blue), 8 (green), and 24 (red) $\mu \mathrm{m}$ illustrate the wide variation of star-formation activity configurations as compared to the stellar distribution in spiral galaxies. The galaxies are, clockwise from upper left-hand corner, NGC628 SAc, NGC3031 = M81 SAab, NGC3521 SABbc, NGC3184 SABcd, NGC4725 SABab, NGC3627 SABb, NGC3351 SBb, and NGC1097 SBb.

Explorer (GALEX) data, show that IR-warm galaxies have a large scatter in their IR/UV ratio, whereas galaxies with cooler dust show less dispersion.

Spitzer imaging data refined ISO results (cf. Sauvage et al. 2005), tracing spiral arms and starformation regions with greater resolution. They showed that star-formation regions are more compact at $24 \mu \mathrm{m}$ than at $8 \mu \mathrm{m}$, confirming that the latter traces aromatic compounds in photodissociation regions (PDRs) surrounding the HII regions, whereas $24-\mu \mathrm{m}$ emission is more intimately associated with the HII regions themselves (Helou et al. 2004). This phenomenon is also observed in the Milky Way by Spitzer (Churchwell et al. 2006) and by the Midcourse Space Experiment (MSX) (Kraemer et al. 2003). These results revealed in individual cases striking interstellar medium configurations that were unsuspected from other images (Pahre et al. 2004b, Bendo et al. 2006, Gordon et al. 2006).

4.1.2. Spectroscopic studies. IRS spectroscopy of low-metallicity objects yielded intriguing spectra, such as SBS0335-052 where Houck et al. (2004) found silicate absorption (following a preliminary identification of silicates from the ISO spectrum of Thuan, Sauvage \& Madden 1999), no aromatic emission, and the thermal dust continuum peaking near $28 \mu \mathrm{m}$, implying little dust at $\mathrm{T}<100 \mathrm{~K}$. On the other hand, the similarly metal-deficient I $\mathrm{Zw} 18$ (Wu et al. 2007) shows a steeply rising spectrum toward $70 \mu \mathrm{m}$, and again no aromatic features. Engelbracht 


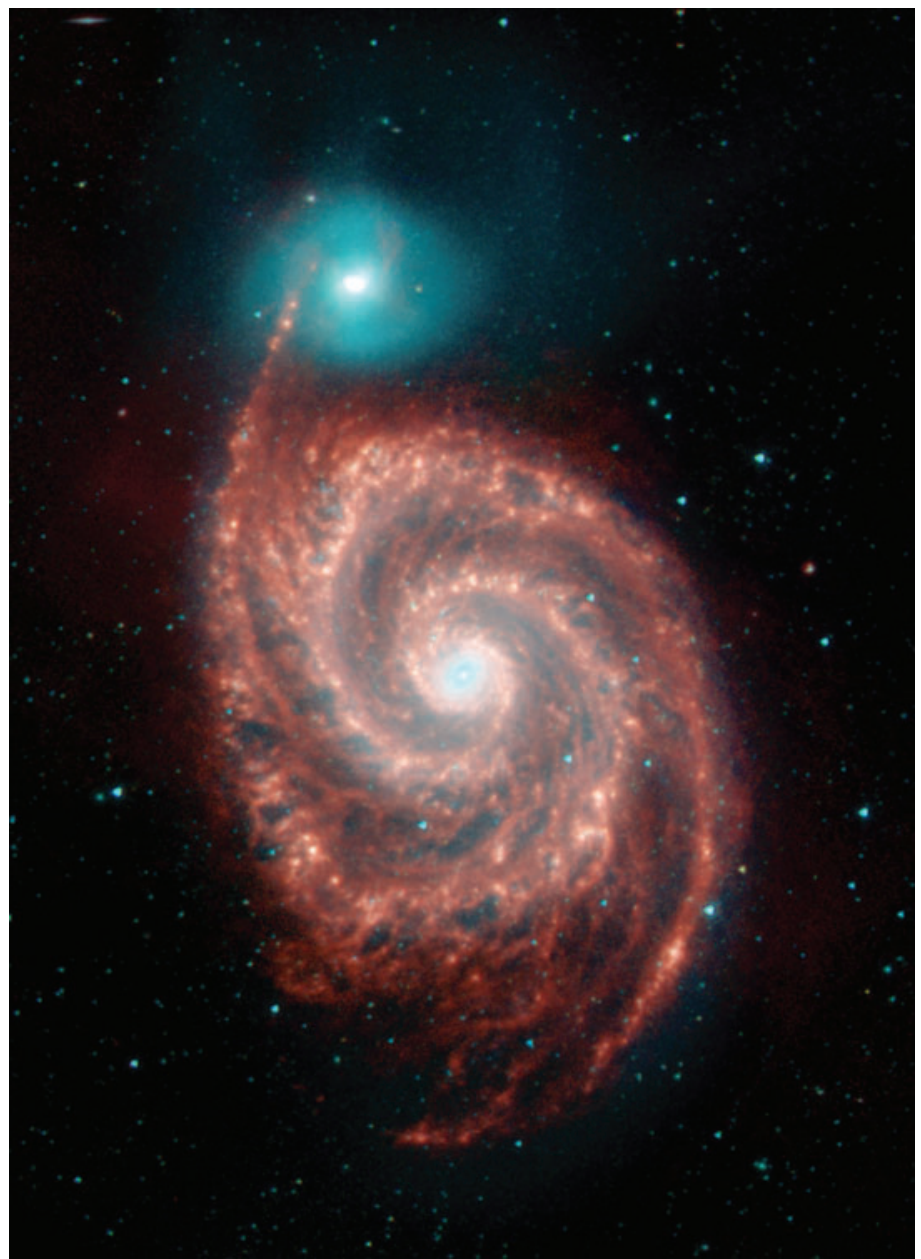

Figure 10

Messier 51a/b as imaged by Spitzer at 3.6 (blue), 4.5 (green), 5.8 (orange), and 8 (red) $\mu \mathrm{m}$. Note that the companion M51b has almost purely stellar colors, whereas the aromatic emission covers the whole disk of M51a, including intricate structures in the interarm regions.

et al. (2005) used broad-band 3.6-24- 4 m SEDS in 34 objects to argue for a significant drop in aromatic feature emission once metallicity drops below $1 / 3$ to $1 / 5$ solar. Bolatto et al. (2007) invoke photodestruction of aromatic compounds to explain the $8-\mu \mathrm{m}$ deficiency in the SMC, whereas Jackson et al. (2006) interpret the paucity of $8-\mu \mathrm{m}$ emission in low-metallicity dwarf galaxies with an older stellar population as evidence for destruction by supernova shocks. O'Halloran, Satyapal \& Dudik et al. (2006) cite the $[\mathrm{Fe} \mathrm{II}] /[\mathrm{Ne} \mathrm{II}]$ rise with decreasing metallicity as further evidence of this destruction process. It would seem then that aromatic deficiency is indeed associated with low metallicity, but that the general shape of the continuum and its peak vary widely among irregular galaxies, possibly depending on the phase of star formation activity during which each of these volatile systems is observed (Higdon, Higdon \& Marshall 2006, Hinz et al. 2006).

Determining the SEDs of galaxies constrains models of dust populations and their heating within galaxies (Dale et al. 2005, Wen et al. 2007). Spitzer has largely confirmed the general 
validity of pre-Spitzer SED models based on IRAS and ISO data (e.g., Dale \& Helou 2002) that point to $24-\mu \mathrm{m}$ flux as a key discriminant of dust heating in a galaxy, and its association with star formation. Careful SED fitting with sufficiently detailed physical dust models yields more reliable estimates of the dust mass, along with aromatic carrier fractions and heating intensities (Draine et al. 2007). These estimates show that there is no need for cold dust ( $\mathrm{T} \leq 10 \mathrm{~K}$ ) even to account for $850-\mu \mathrm{m}$ fluxes and that for most spiral galaxies dust in the diffuse interstellar medium (ISM) dominates the IR luminosity. Draine et al. also find that there is a positive dependence of dust-to-gas ratio on metallicity $\mathrm{A}_{0}=12+\log (\mathrm{O} / \mathrm{H})$, and the aromatic carrier fraction drops appreciably when $\mathrm{A}_{0}$ drops below 8.1. It remains the case, however, that aromatic feature strength is not a simple linear function of metallicity (Rosenberg et al. 2006, Walter et al. 2007).

Brandl et al. (2006) found substantial variation in the SEDs of starbursts in several key attributes such as the depth of silicate absorption, aromatic feature equivalent width, and 15- to $30-\mu \mathrm{m}$ continuum slope. The expected correlations, for instance between continuum slope and $[\mathrm{Ne} \mathrm{III}] /[\mathrm{Ne} \mathrm{II}]$ ratio, appear to be lost because of aperture averaging, which dilutes the starburst nucleus itself with varying amounts of disk contributions.

\subsection{The High-Intensity and Exotic Universe}

Since the IRAS survey much observational effort has been devoted to detailed studies of high luminosity, infrared-bright galaxies. Spitzer has followed this with particular emphasis on spectroscopic studies enabled by the broad spectral coverage and enormous sensitivity gain of the IRS.

4.2.1. Local ULIRGs. ULIRGs were originally identified individually from ground-based IR photometry by Rieke \& Low (1972), and from early analysis of IRAS survey data (Soifer et al. 1984), which found a number of galaxies with enormous far-IR luminosities and very large fractions $(>90 \%)$ of their bolometric luminosities emerging in the far-IR. With the IRAS all-sky survey, ULIRGs were identified as a class of highly interesting but locally rare systems (Sanders et al. 1988, see Sanders \& Mirabel 1996 for a summary), with bolometric luminosities matching those of quasars and a space density equivalent to that of local UV-selected quasars (Soifer et al. 1987).

Because the luminosities and space densities of ULIRGs were well matched to local quasars, the prime question that drove much ULIRG research was "what powers the luminosity?," with both dust-enshrouded AGN (Sanders et al. 1988) and starburst models (Heckman 1994) finding strong support from different observations. Observations with ISO made great advances in studying these systems and in understanding the ULIRGs (see Genzel \& Cesarsky 2000 for a comprehensive review).

A major step forward from the ISO studies (Genzel et al. 1998, Laurent et al. 2000) was the recognition that the strength of the aromatic feature emission was strongly correlated with the origin of activity, with strong aromatic emission being found in starburst systems and weak emission being associated with systems identified as Seyfert 1 nuclei and quasars. ISO studies showed that at $L \sim 10^{12} L_{\odot}$ most ULIRGs are apparently powered predominantly by star formation, but for luminosities $>3 \times 10^{12} L_{\odot}$ the dominant luminosity source appears to become a dustenshrouded AGN. One great difficulty with understanding ULIRGs is that different diagnostics lead to different assessments of what is the most important power source (cf. Soifer et al. 2000).

The greater Spitzer sensitivity for spectroscopy has led to more extensive and more detailed studies of ULIRGs in the local universe $(z<0.5)$ and the extension of the mid-IR diagnostics to substantially higher redshifts $(z>2)$. Armus et al. $(2006,2007)$ have reported both low- and high-resolution IRS spectra of 10 bright ULIRGs at $z<0.1$ and NGC 6240 over the full wavelength range accessible to IRS. Farrah et al. (2007) have reported results from high-resolution 
spectroscopy of 53 ULIRGS with $z<0.32$, while Imanishi et al. (2007) have reported 5-35- $\mu \mathrm{m}$ low-resolution spectra of 48 ULIRGS classified from optical spectroscopy as either low-ionization nuclear emission-line regions (LINERs) or HII region systems at $z<0.15$.

Armus et al. (2006) found that NGC 6240 has [NeV] 14.3- $\mu$ m emission, requiring photons of energy $\mathrm{E}>97 \mathrm{eV}$ to create $\mathrm{Ne}^{4+}$, presumably associated with an AGN. After correcting for extinction to the $\mathrm{Ne}^{4+}$ region from the AGN, Armus et al. find that $\sim 20 \%$ of the bolometric luminosity of NGC 6240 is powered by an AGN, consistent with analysis of the X-ray data.

Armus et al. (2007) find that there is no "characteristic" ULIRG spectrum, but rather the low-resolution spectra (Figure 11) range from nearly featureless, with weak silicate absorption dominating in Markarian 231, to extremely deep silicate absorption (IRAS 08572+3915). Variations in silicate absorption and aromatic feature emission lead to great diversity in IR spectra. In addition, Armus et al. find absorption associated with $\mathrm{C}_{2} \mathrm{H}_{2}$ and $\mathrm{HCN}$ molecules in 4 out of 10 ULIRGs and water ice absorption is found in 7 out of 10 ULIRGs. Consistent with the work of Genzel et al. (1998), Armus et al. find that the $[\mathrm{NeV}] /[\mathrm{NeII}]$ line ratio decreases with increasing $6.2-\mu \mathrm{m}$ equivalent width, suggesting that as the UV radiation field softens, the relative strength of the aromatic feature increases.

The high-resolution ULIRG spectra commonly reveal emission from forbidden lines of the ions $\mathrm{Ar}^{2+}, \mathrm{Ne}^{+}, \mathrm{Ne}^{2+}, \mathrm{Ne}^{4+}, \mathrm{S}^{+}, \mathrm{S}^{2+}, \mathrm{S}^{3+}, \mathrm{Si}^{+}$, and $\mathrm{O}^{3+}$, with lines of $\mathrm{Ar}^{4+}, \mathrm{Cl}^{+}, \mathrm{P}^{2+}, \mathrm{Fe}^{+}$, and $\mathrm{Fe}^{2+}$ seen occasionally. The $[\mathrm{NeV}]$ line is found in 3 out of 10 of the BGS ULIRGs. Farrah et al. (2007) detect $[\mathrm{NeV}]$ emission in just under half of their sample of 53 ULIRGs, indicating that an AGN is present (though not necessarily dominant) in this fraction of the sample. They find from the strength of the $[\mathrm{NeV}]$ line that an AGN is the dominant power source in $\sim 20 \%$ of the ULIRGs. In contradiction to previous studies, they do not find a correlation of AGN fraction with ULIRG luminosity.

Desai et al. (2007) find a 2-order-of-magnitude range $(0.006-0.8 \mu \mathrm{m})$ in the equivalent width of the $6.2-\mu \mathrm{m}$ band in low-resolution spectra of 103 ULIRGs, and they attribute this range to variation of the underlying continuum emission associated with hot dust, rather than extinction or destruction of the aromatic molecules. For starburst ULIRGs they find the average equivalent width of the 6.2- $\mu \mathrm{m}$ feature to be half that of lower-luminosity starbursts (Brandl et al. 2006). Most of the ULIRGs with suppressed $6.2-\mu \mathrm{m}$ emission have high silicate absorption at $9.7 \mu \mathrm{m}$, and the equivalent widths of both the 6.2 - and $11.3-\mu \mathrm{m}$ bands decrease with increasing $25-\mu \mathrm{m} / 60-\mu \mathrm{m}$ flux ratio, and also with increasing $v L_{v}(24 \mu \mathrm{m})$.

Contradictory results are reported by Imanishi et al. (2007) based on 5- to 35- $\mu \mathrm{m}$ spectra of 48 ULIRGs at $z<0.15$. In agreement with Desai et al. they find that in 40-50\% of their sample the aromatic features are substantially weaker (in equivalent width) than for starburst systems of lower luminosities. They argue that this is due to a highly obscured, compact AGN luminosity source. With $40-50 \%$ of their sample showing some evidence of a dust-enshrouded AGN, and $1 / 3$ of the optically classified non-Seyfert ULIRGs showing strong signatures of buried AGN, they argue that in total, including the ULIRGs that are classified optically as AGN, more than $50 \%$ and possibly more than two-thirds of ULIRGs within $z<0.15$ have a luminous AGN.

Luminosity alone is an unreliable indicator of the energy source. MIPS J142824.0+352619 (Borys et al. 2006) is a galaxy with an apparent $L_{\mathrm{ir}} \sim 3.2 \times 10^{13} L_{\odot}$, possibly amplified by less than a factor of 10 by a foreground lens. It is unusual in revealing no trace of an AGN and is therefore among the most luminous pure starbursts known, possibly a "low-redshift" analog of the SMGs.

Spoon et al. (2006) have identified absorption by crystalline silicates in 12 ULIRGs with a fraction of crystalline to amorphous silicates of 0.11 , or 12 times the upper limit to the contribution of crystalline silicates to the ISM in the Milky Way. They argue that because of the high starformation rates, the timescale for injection of crystalline silicates is substantially shorter in these 


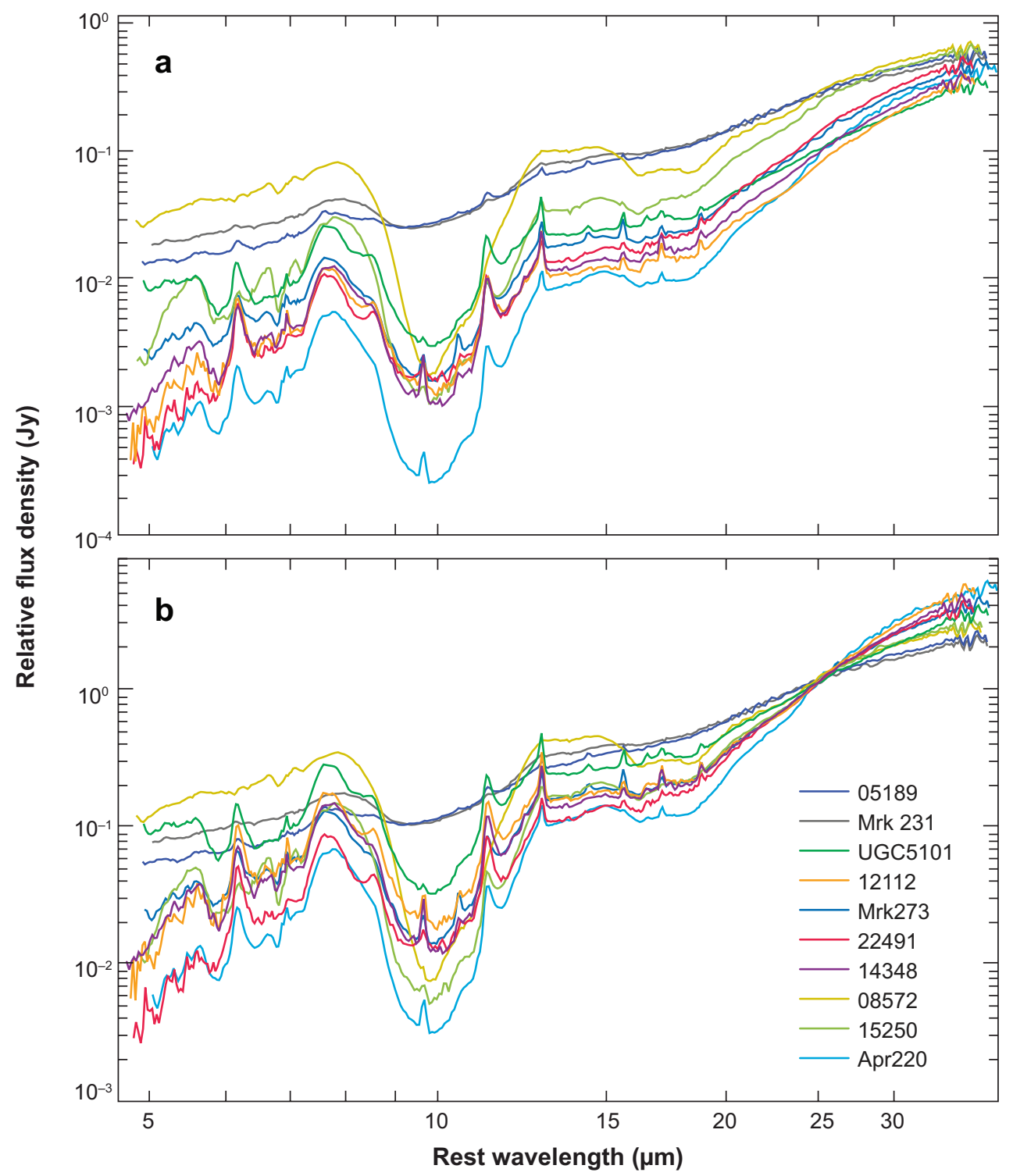

Figure 11

Spectral energy distribution of 10 ULIRGs selected from the IRAS Bright Galaxy Sample (Armus et al. 2006, figure used by permission). (a) The spectra normalized at the rest frame $60-\mu \mathrm{m}$ point. (b) The spectra normalized at the rest frame $24-\mu \mathrm{m}$ point. Note the substantial dispersion in slope, silicate absorption depth, and prominence of aromatic feature emission.

ULIRGs than in the Milky Way, whereas the timescale for "amorphization" is no longer than the $10^{8}$ years found in the Milky Way.

Spoon et al. (2007) plot the equivalent width of the 6.2- $\mu \mathrm{m}$ emission band versus the strength of the $9.7-\mu \mathrm{m}$ silicate band for a wide variety of objects ranging from ULIRGs to AGN to starburst systems (Figure 12), finding two main tracks, one with relatively constant, small silicate strength (weak emission or absorption) and a wide range of $6.2-\mu \mathrm{m}$ equivalent width, and a "diagonal" 


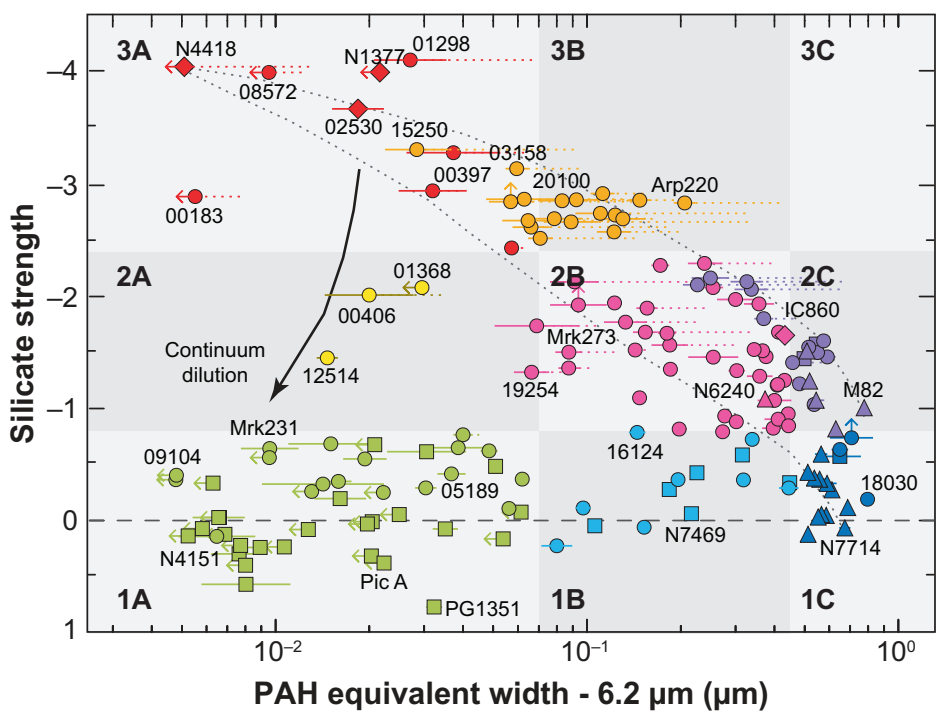

Figure 12

Diagnostic plot of the equivalent width of the 6.2- $\mu \mathrm{m}$ PAH emission feature versus the 9.7- $\mu \mathrm{m}$ silicate strength. Upper and lower limits are indicated by arrows. Galaxy types are distinguished by their plotting symbol: filled circles, ULIRGs and HyLIRGs; filled triangles, starburst galaxies; filled squares, Seyfert galaxies and QSOs; filled diamonds, other IR galaxies. See Spoon et al. (2007) for further details (figure used by permission).

track, where the $6.2-\mu \mathrm{m}$ band strength decreases with increasing silicate absorption strength. They suggest that the track of weak, optically thin silicate emission/absorption is the transition from AGN to starburst dominance as the 6.2- $\mu \mathrm{m}$ aromatic band strength increases. The AGN portion of this track is associated with a clumpy dust distribution. The diagonal track, dominated by geometric effects where the deeper silicates (and weaker 6.2- $\mu \mathrm{m}$ emission) correspond to dust that is geometrically smooth becoming optically thick, is where nearly all the ULIRGs are found. The geometric interpretation of the differing mid-IR spectra follows the models of Levenson et al. (2007), and is broadly consistent with the interpretation by Sajina et al. (2007).

4.2.2. ULIRGs at high redshifts. In the nearby universe, ULIRGs are defined by $L_{\mathrm{bol}}>10^{12} L_{\odot}$, with the vast majority of this luminosity emerging at wavelengths beyond $5 \mu \mathrm{m}$. The local luminosity function is $n(L) \sim L^{-2}$ (cf. Soifer et al. 1987, Sanders et al. 2003), so the number densities drop steeply with luminosity and there are very few ULIRGs locally with $L>3 \times 10^{12} L_{\odot}$. It is believed that locally for $L<3 \times 10^{12} L_{\odot}$, starbursts dominate the energetics of ULIRGs (see above), but at higher luminosities AGN become the predominant power sources.

Several groups have used 24- $\mu \mathrm{m}$ fluxes in combination with shorter-wavelength Spitzer observations and/or visible fluxes to select objects likely to be high-luminosity objects at high redshift. Houck et al. (2005) present IRS spectra of 17 out of 30 sources selected from the Boötes MIPS survey to have $f(24 \mu \mathrm{m})>0.8 \mathrm{mJy}$ and $v f_{v}(24 \mu \mathrm{m}) / v f_{v}(0.7 \mu \mathrm{m})>100$ (i.e., R-[24] $>15 \mathrm{mag}$ ). All 17 of these sources have redshifts in the range of $1.7<z<2.8$ and are thus extremely luminous. Houck et al. argue from the dominance of silicate absorption in these sources that they are predominantly AGN-powered.

L. Yan et al. (2007) have selected high-redshift ULIRGs with $v f_{v}(24 \mu \mathrm{m}) / v f_{v}(0.7 \mu \mathrm{m})>$ 10 , and $v f_{v}(24 \mu \mathrm{m}) / v f_{v}(8.0 \mu \mathrm{m})>3$. In their sample, 35 out of 47 sources have $1.5<z<3.2$; 
one-third show strong aromatic features, one-third have only silicate absorption, and the remaining sources have an almost featureless continuum. Sajina et al. (2007) perform more detailed analysis of spectra and SEDs of this sample, finding that three-fourths of the sources are dominated by smooth continua, suggesting an AGN origin to the bulk of the luminosity, but $60 \%$ of the sample have PAH features, arguing for a measurable contribution to the luminosity from star formation.

Teplitz et al. (2007) obtained the $21-37 \mu \mathrm{m}$ spectrum of a $z=2.69$ object with a $24-\mu \mathrm{m}$ flux of only $0.15 \mathrm{mJy}$. Rigby et al. (2008) have obtained spectra of strongly lensed galaxies at $z>2$. These spectra show that the aromatic spectrum is little changed from $z>2.5$ to the local universe. The strong aromatic emission in these objects reveals that this constituent of the interstellar medium was in place just a few gigayears after the Big Bang.

With few high-redshift ULIRGs detected at $\lambda>24 \mu \mathrm{m}$, it is difficult to establish their bolometric luminosities. None of the sources in the Houck et al. sample was detected at wavelengths greater than $24 \mu \mathrm{m}$ in the MIPS Boötes survey (although some were detected at 70 and $160 \mu \mathrm{m}$ in follow-on pointed observations; Tyler et al. 2008). The range of bolometric luminosities for the sources in the Houck et al. sample was estimated to be $0.5-5 \times 10^{13} L_{\odot}$, but the bolometric corrections (from $v L_{v}$ at the observed wavelengths to $L_{\mathrm{bol}}$ ) are highly uncertain, ranging from factors of 5 to 30 based on the assumed SEDs.

L. Yan et al. (2005b) estimate a bolometric luminosity of $\sim 2 \times 10^{13} L_{\odot}$ for one source with strong aromatic emission based on detections at $70 \mu \mathrm{m}$ and $1.2 \mathrm{~mm}$ with an uncertainty of a factor of 2-3. They attribute the majority of this luminosity to star formation. Sajina et al. (2007) argue that the luminosities of L. Yan et al. selected ULIRGs are 5-10 times greater than ULIRGs in the local universe, and they find a handful of ULIRGs with strong aromatic features and $v L_{v}(14 \mu \mathrm{m})>10^{12} L_{\odot}$, suggesting that starburst galaxies at high redshifts reach bolometric luminosities of more than $10^{13} L_{\odot}$.

However, Brand et al. (2007) argued for the importance of AGN in the most luminous ULIRGs by studying 10 very red (R-[24] $>14 \mathrm{mag})$, extremely luminous $\left(>3 \times 10^{12} L_{\odot}\right)$ galaxies at $z \sim$ 2 in Boötes. Ground-based near-IR spectra (rest-frame visible) show in every case either broad emission lines or emission line ratios, indicating that they are AGN-dominated. Both the Type I and Type II AGN in this sample show considerable evidence of extinction.

4.2.2.1. ULIRGs selected at $24 \mu m$ versus other means. Samples of ULIRGs, by definition IR-selected, generally do not match those from visible/near-IR methods of identifying distant, luminous galaxies (e.g., LBG, BzK, or DRG samples), and generally ULIRGs at $z>1.5$ are invisible in the deepest ground-based imaging at wavelengths shorter than $\mathrm{R}$ band. Dey et al. (2008) argue that systems with R-[24] > 14 represent a class of galaxies that have been missed in deep optical imaging. Their study has shown these systems have $\langle z\rangle \sim 2$ with $\sigma_{\mathrm{z}} \sim 0.5$, and although the apparent surface density is comparatively low $\left[\sim 0.09(\mathrm{sq} \operatorname{arcmin})^{-1}\right.$ at $f_{v}(24 \mu \mathrm{m})>$ $0.3 \mathrm{mJy}]$, the high bolometric luminosities compensate to some extent and thereby provide an IR luminosity per unit volume nearly equivalent to that produced by the IR-luminous systems found by other means.

Alternatively, submillimeter-selected and 24- $\mu$ m-selected samples in the $z \sim 2$ range are both ULIRG populations with substantial overlap, but also with distinct selection biases. The $24-\mu \mathrm{m}-$ selected sources tend to harbor AGN, which boost their mid-IR featureless continuum emission, whereas SMGs favor star formation as the dominant or only power source, which leads to high aromatic ratios, low mid-IR continuum, and cooler emission in the far-IR (see Section 3.3.2).

4.2.3. QSOs and unification theories. Although deep silicate absorption at $10 \mu \mathrm{m}$ is commonly seen in IR-luminous AGN, the corresponding emission feature had not been found prior to Spitzer. 
Unified models attribute the differences between Type I and Type II AGN to differences in the observer's vantage point. Type I AGN are viewed along the polar axis of an accretion disk or molecular torus, where silicates should be seen in emission, while Type II objects are seen in the plane of the disk, with resulting silicate absorption from viewing the hot inner regions of the torus through the outer cold material. Siebenmorgen et al. (2005) and Hao et al. (2005) have reported Spitzer detections of silicate emission that provide confirmation of this prediction of the unified model. In particular, Hao et al. observed both the 10- and the 18- $\mu \mathrm{m}$ Si features in five low-redshift PG quasars. The emission spectra that they observe can be reasonably well fit by standard grain models based primarily on amorphous silicates. Interestingly, the ratio of the silicate emission in the two bands corresponds to grain temperatures around $\sim 200 \mathrm{~K}$, much lower than the sublimation temperature $>1000 \mathrm{~K}$, which one might expect of dust in the inner regions of the torus.

Shi et al. (2006) have compiled IRS spectra of almost 100 AGN that exhibit a range of silicate optical depths in both emission and absorption. They show that the silicate optical depth correlates well with the absorbing $\mathrm{HI}$ column density inferred from the $\mathrm{X}$-ray data on these sources. The $\mathrm{HI}$ column density varies from $1 \times 10^{20}$ to more than $1 \times 10^{24} \mathrm{~cm}^{-2}$. The results are consistent with a picture in which, on average, a source showing strong silicate absorption shows a large HI column density; for objects with column densities much below $1 \times 10^{22} \mathrm{~cm}^{-2}$ the silicate feature is seen in emission, with an inverse correlation between feature strength (relative to the continuum) and column density. Shi et al. present a notional model of the distribution of material around the central accretion disk that accounts qualitatively for both the average relationship and the considerable scatter around it. The main determinants of the appearance of a spectrum are the line of sight along which the structure is viewed and the number of clouds or clumps of material that the line of sight intercepts.

Mid-IR spectra of UV-selected quasars show that these quasars have clear diagnostics of high excitation emission lines from $\mathrm{Ne}^{4+}$ and $\mathrm{Ne}^{5+}$, as well as weak aromatic emission (Schweitzer et al. 2006, Netzer et al. 2007, Shi et al. 2007). They show a good correlation between the 7.7- $\mu \mathrm{m}$ aromatic emission and the FIR continuum at $60 \mu \mathrm{m}$, and suggest that the FIR emission is associated with star formation (that is producing the 7.7- $\mu \mathrm{m}$ emission). They suggest that the far-IR contin$\operatorname{uum}(>50 \mu \mathrm{m})$ in UV-selected quasars is due to a circumnuclear starburst that can be as luminous as that of starburst-dominated ULIRGs in the local universe. Richards et al. (2006) characterized the diversity in SEDs among Type 1 QSOs, using 259 objects with Spitzer and Sloan data, and showed that a mean SED can estimate the bolometric luminosities of individual quasars to within $50 \%$.

Lacy et al. (2004) and Stern et al. (2005) have utilized the combination of IRAC imaging and SDSS data to define mid-IR color criteria to identify AGN. The selection is based purely on IRAC colors. Stern et al. find their color selection to be $80 \%$ complete and less than $20 \%$ contaminated when compared with optical spectroscopic criteria. Finally, Jiang et al. (2006) have studied $13 z \sim$ 6 quasars from 3.6 to $24 \mu \mathrm{m}$ and find SEDs generally are similar to those of low-redshift quasars at the same rest-frame wavelengths, suggesting that these distant objects have reached maturity $\sim 1$ Gyr after the Big Bang.

\subsection{The Astrophysics of IR Emission}

Spitzer data, often combined with data from other facilities, have been analyzed to reveal the physical conditions in the ISM of galaxies. They have been used to identify trends relating properties that reflect causal connections or underlying physical phenomena.

4.3.1. Aromatic features and molecular hydrogen. Smith et al. (2007) studied in detail the behavior of mid-IR spectra of the central regions of SINGS (see sidebar) galaxies selected to be 
dominated by star formation though occasionally harboring an AGN. They found that silicate absorption was detectable in only $\sim 1$ out of 8 of the sample, and that ratios among aromatic features varied by factors of 2 to 5, driven primarily by the presence of an AGN and secondarily by metallicity. The aromatic features carry typically $7 \%$ to $15 \%$ of the IR luminosity, though this fraction ranges from less than $2 \%$ to almost $20 \%$. The fraction of IR luminosity carried by the aromatic features covers roughly the same range whether or not the galaxy harbors an AGN, though the presence of the latter biases the fraction toward lower values.

The pure rotational transitions of molecular hydrogen are valuable as a direct measure of temperatures and masses of the most abundant molecule, $\mathrm{H}_{2}$. ISO studied the rotational $\mathrm{H}_{2}$ lines in a variety of Milky Way objects, demonstrating this promise, but detected them in only two normal galaxies (cf. Sauvage et al. 2005), and a few active galaxies (cf. Verma et al. 2005). Spitzer is providing a much fuller picture of $\mathrm{H}_{2}$ in galaxies. Roussel et al. (2007) studied emission in the rotational transitions of $\mathrm{H}_{2}$ from kiloparsec-scale areas in the centers of SINGS galaxies. The lowest-energy transitions $\mathrm{S}(0) 28.22 \mu \mathrm{m}, \mathrm{S}(1) 17.04 \mu \mathrm{m}, \mathrm{S}(2) 12.28 \mu \mathrm{m}$, and $\mathrm{S}(3) 9.67 \mu \mathrm{m}$ are readily detected in a majority of SINGS galaxies, the $\mathrm{S}(1)$ line being most luminous and detected in $86 \%$ of SINGS galaxies. The $\mathrm{H}_{2}$ detected by Spitzer is excited to $\gtrsim 100 \mathrm{~K}$ and is a substantial fraction (1-30\%) of the total $\mathrm{H}_{2}$ column estimated from CO data. The $\mathrm{S}(0), \mathrm{S}(1)$, and $\mathrm{S}(2)$ lines together carry typically $4 \times 10^{-4} L_{\mathrm{ir}}$ from the same area of the galaxy, which is roughly an order of magnitude below the major fine-structure lines of [CII] and [OI] (Malhotra et al. 2001) and a factor of 3 below the lines of [SiII]. The luminosity of $\mathrm{H}_{2}$ lines tracks the aromatic feature luminosity with a smaller dispersion ( $0.1 \mathrm{dex})$ than $L_{\mathrm{ir}}(0.15 \mathrm{dex})$ or $L_{24} \mu \mathrm{m}$ ( $\left.0.28 \mathrm{dex}\right)$, pointing to a close connection between the power sources of aromatics and warm $\mathrm{H}_{2}$. Galaxies with AGNs (Seyfert or LINER signature in the visible spectra) display excess $\mathrm{H}_{2}$ emission attributed to shocks. Roussel et al. suggested that galaxies with $L\left(\mathrm{H}_{2}\right.$ lines $) / L$ (aromatics) $>1.5 \%$ have a low-luminosity AGN. Brunner et al. (2008) mapped M51 in the $\mathrm{S}(0)$ to $\mathrm{S}(5) \mathrm{H}_{2}$ lines, finding a warm phase $\left(\mathrm{T}_{\mathrm{exc}}<300 \mathrm{~K}\right)$ mostly coincident with the dust lanes, and a hot phase $\left(\mathrm{T}_{\text {exc }}>400 \mathrm{~K}\right)$ mostly in the nuclear region, coincident with [OIV] and X-ray emission, suggesting that shocks or X-rays are responsible for its excitation.

Many ULIRGs show strong emission lines of $\mathrm{H}_{2}$. Armus et al. (2006) detected the $\mathrm{S}(0)$ to $\mathrm{S}(7)$ lines of $\mathrm{H}_{2}$ [except the $\mathrm{S}(6)$ line, which is blended with the $6.2-\mu \mathrm{m}$ aromatic feature at the resolution of the IRS] in NGC 6240. From these lines they find $10^{7} M_{\odot}$ of hot $(950 \mathrm{~K})$ and $1.6 \times 10^{9} M_{\odot}$ of warm $(160 \mathrm{~K}) \mathrm{H}_{2}$. This represents $15 \%$ of the total $\mathrm{H}_{2}$ as measured by singlebeam $\mathrm{CO}$ observations and $50 \%$ of the gas within $1 \mathrm{kpc}\left(2^{\prime \prime}\right)$ of the nucleus of this interacting system. Higdon et al. (2006) analyze the $\mathrm{H}_{2}$ spectra of 77 ULIRGs, including the 10 BGS ULIRGs observed by Armus et al. (2007). They find that $\mathrm{H}_{2} \mathrm{~S}(1), \mathrm{S}(2)$, and S(3) lines are detected in $~ 3 / 4$ of their sample, and in all of the ULIRGs with $60-\mu \mathrm{m}$ flux density $>2 \mathrm{Jy}$. Of the ULIRGS with $\mathrm{H}_{2}$ emission, a majority are fitted with a single-temperature warm gas with $\left\langle\mathrm{T}_{\text {exc }}\right\rangle=336 \pm 15$ $\mathrm{K}$ and an average mass of $\left\langle M_{\mathrm{H}_{2}}\right\rangle=2 \times 10^{8} M_{\odot}$. Hot $\mathrm{H}_{2}$ is found in six ULIRGs, with $\left\langle\mathrm{T}_{\text {hot }}\right\rangle \sim$ $1400 \mathrm{~K}$ and $\left\langle M_{\mathrm{hot}}\right\rangle \sim 3 \times 10^{6} M_{\odot}$. Higdon et al. find that the median ratio of $\mathrm{H}_{2}$ mass determined from mid-IR emission to that from CO emission is $M_{\mathrm{irs}} / M_{\mathrm{CO}} \sim 0.3 \%$. The $\mathrm{H}_{2}$ line ratios found are consistent with little or no extinction in the mid-IR, suggesting that the warm molecular material lies outside the region of high extinction indicated from the silicate absorption in the ULIRGs.

The intergalactic shock in Stephan's Quintet revealed a remarkable mid-IR spectrum dominated by $\mathrm{H}_{2}$-line emission extending over $24 \mathrm{kpc}$, and carrying 10 times the surface luminosity of soft X-rays and one-third of the total IR luminosity (Appleton et al. 2006); this unexpected result illustrates the importance of shocks in $\mathrm{H}_{2}$ excitation and the potential of $\mathrm{H}_{2}$ as a major coolant of star-forming gas in the early universe. In a possibly related phenomenon, Egami et al. 
(2006) report exceptionally strong $\mathrm{H}_{2}$ lines from an IR-luminous, brightest cluster galaxy in the $\mathrm{X}$-ray-luminous cluster Zwicky 3146. Although the geometry is harder to pin down at $z=0.29$, it is entirely plausible that shocks in cooling flows are again responsible for the prominence of the $\mathrm{H}_{2}$ lines. Ogle et al. (2007) also report that the Spitzer spectrum of the giant FR II radio galaxy $3 \mathrm{C} 326$ is dominated by $\mathrm{H}_{2}$ emission, with the $\mathrm{H}_{2}$ lines contributing $17 \%$ of the 8 - to $70-\mu \mathrm{m}$ luminosity. They attribute the emission to shock heating in an accretion flow, and point out that $\mathrm{H}_{2}$ may dominate the cooling of infalling gas, thus allowing the gas to settle and start feeding both starburst activity and black hole accretion.

Adding to the connection between low-excitation transitions and AGN, Dale et al. (2006) showed that mid-IR lines of low-ionization species such as [Si II], bracketed between ionization energies of 8.15 and $16.35 \mathrm{eV}$, provide efficient discrimination between star formation and Seyfert or LINER power sources. When [Si II] is enhanced, the reliability of association with a Seyfert or LINER is at least $90 \%$, presumably because of the importance of $\mathrm{X}$-ray-illuminated regions (e.g., Maloney, Hollenbach \& Tielens 1996) in AGN-powered systems.

We plot in Figure 13 the ratio of $\mathrm{H}_{2}$-line luminosity-summing over the three transitions S(1), S(2), and S(3) - to total IR luminosity, as a function of total IR luminosity for a compilation of nearby galaxies (Roussel et al. 2007), nearby ULIRGs (Higdon et al. 2006), and a number of other objects. The upper part of the plot reflects enhanced $\mathrm{H}_{2}$ emission owing to shock excitation. ULIRGs, however, are clearly lower than, and almost disjoint from, star-forming galaxies in their $\mathrm{H}_{2} / L_{\mathrm{ir}}$ ratios and much lower than pure shocked gas regions. The $\mathrm{H}_{2} / L_{\mathrm{ir}}$ ratio may be useful as an empirical discriminator between low-density, disk-like systems and high-density, high-intensity systems like ULIRGs.

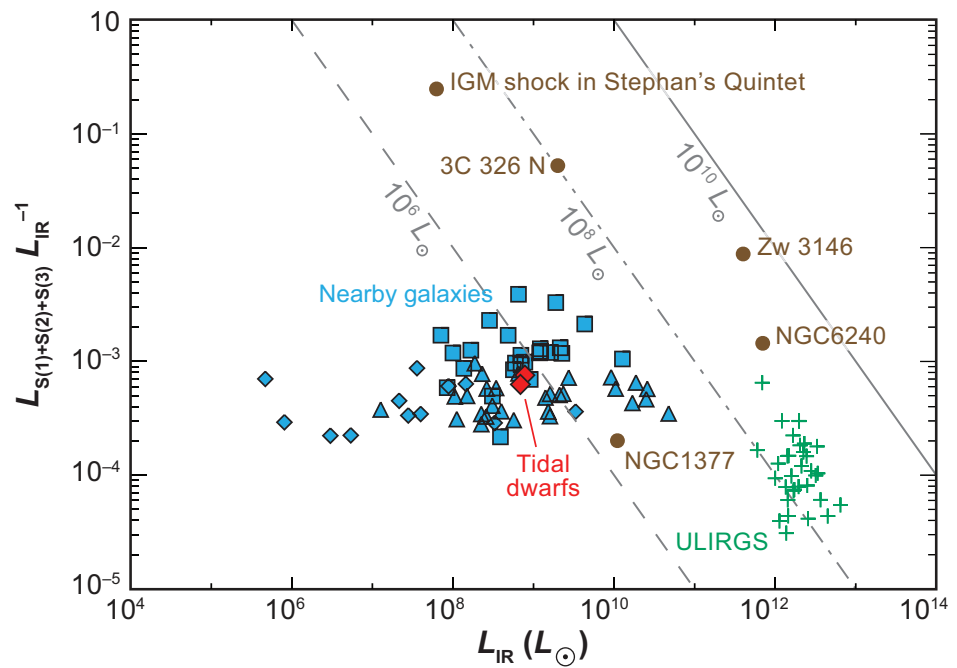

Figure 13

Compilation of $\mathrm{H}_{2}$ measurements for normal galaxies (Roussel et al. 2007), ULIRGs (Higdon et al. 2006), and a variety of other objects. The ratio of the luminosity in the sum of the $S(1), S(2)$ and $S(3)$ lines (of the ground vibration state) of $\mathrm{H}_{2}$ to the IR luminosity of the system is plotted versus the IR luminosity. Nearby galaxies are shown as filled blue symbols; diamonds represent dwarf galaxies, triangles represent star-forming galaxies with no other type of activity, and squares represent galaxies with Seyfert or LINER nuclei.

ULIRGs are shown as crosses. Gray lines of constant $\mathrm{H}_{2}$ luminosity $\left(10^{6}, 10^{8}\right.$, and $\left.10^{10} L_{\odot}\right)$ show that, in spite of the variations in $L_{\mathrm{H}_{2}} / L_{\mathrm{ir}}$, ULIRGs are still more luminous in $\mathrm{H}_{2}$ rotational lines, whereas intergalactic shocks can easily outshine whole galaxies in these lines. 
4.3.2. Other molecular tracers in ULIRGs. Lahuis et al. (2007) have detected absorption due to $\mathrm{C}_{2} \mathrm{H}_{2}$ and $\mathrm{HCN}$ in 15 IR-luminous galaxies, 13 of which are ULIRGs. They also detect $\mathrm{CO}_{2}$ absorption in four systems, three of which are ULIRGs. They argue that these detections establish the presence of warm $(200-700 \mathrm{~K})$, dense $\left(n_{\mathrm{H} 2}>10^{7} \mathrm{~cm}^{-3}\right)$ molecular gas. They further argue that this gas cannot be $\mathrm{X}$-ray excited because the molecules would be destroyed on too short a timescale. Instead, they suggest that the conditions require highly embedded star formation, where the high pressure and density of the gas have inhibited the disruption of the star-forming molecular cores. From this they conclude that in these cases pressure-confined massive star formation produces the observed $\mathrm{C}_{2} \mathrm{H}_{2}$ and $\mathrm{HCN}$-rich warm gas.

4.3.3. Galaxy-scale processes. Elmegreen et al. (2006) argue that the regularly spaced emission clumps in the interacting galaxies IC 2163 and NGC 2207 point to gravitational instabilities as their origin. Moreover, the IR luminosity function of these clumps is strongly peaked at one value, unlike the optical luminosity function and its power-law structure.

Roussel et al. (2006) combined GALEX and Spitzer data to demonstrate that within NGC 300, the morphologies of HII regions and, by inference, their ages are a key parameter in determining the UV-to-IR flux ratios for the regions. Dale et al. (2007) found that clumpiness at $24 \mu \mathrm{m}$ is directly connected to dust temperatures in the far-IR and to the UV/IR ratio in the whole SINGS sample. They find that irregular galaxies are clumpier, warmer, and have less UV extinction than spirals, suggesting high ISM porosity. In a detailed study of the dwarf irregular galaxies of the M81 group, Walter et al. (2007) found warmer 70/160 $\mu \mathrm{m}$ colors, and dust-to-HI ratios lower by an order of magnitude compared to SINGS spirals, again suggesting dense clumps of dust near young stars and a relatively evacuated medium away from the clumps.

The long-standing search for more reliable SFR measures was given a major boost by the availability of high-quality 24- $\mu \mathrm{m}$ images of galaxies from Spitzer. Kennicutt et al. (2007) linearly combined $\mathrm{H} \alpha$ and 24- $\mu \mathrm{m}$ fluxes to capture in one estimator both the Lyman continuum photons ionizing the HII region and those captured by dust inside and very close to the HII region. This combination proved tightly correlated with the extinction-corrected $\mathrm{H} \alpha$ (using $\mathrm{Pa}-\alpha$ ), and thus a reliable measure of ionizing flux in HII region complexes within M51a. Calzetti et al. (2007; see also Alonso-Herrero et al. 2006) extended this exploration to other SINGS galaxies, as well as local LIRGs, and argued for the validity of either the combination of $24-\mu \mathrm{m}$ and $\mathrm{H} \alpha$ flux or of $24-\mu \mathrm{m}$ flux alone as SFR indicators for HII regions or starbursts. Calzetti et al. also gave calibrations of these indicators and described the conditions for their validity, demonstrating their basic consistency with stellar population models. In spite of the caveats enumerated by Calzetti et al., this is a significant step in quantifying SFRs with a dispersion of $\sim 0.3$ dex. However, aromatic feature fluxes are unlikely to get more accurate than 0.5 dex as SFR indicators, just as they are similarly uncertain indicators of total IR luminosity in the context of high-redshift sources.

Kennicutt et al. (2007) used the $24 \mu \mathrm{m}+\mathrm{H} \alpha$ star-formation index to probe the star-formation law within M51a on scales of 0.5 to $2 \mathrm{kpc}$ and showed the applicability on these scales of the well-established Schmidt power-law relation between SFR surface density and total gas surface density. The slope (1.37 to 1.56$)$ and dispersion $(\sim 1 \mathrm{dex})$ of the relation between these parameters are comparable to values in the disk-averaged Schmidt law (Kennicutt 1998).

The low dispersion $(\sim 0.15 \mathrm{dex})$ in the ratio of far-IR to radio $21-\mathrm{cm}$ flux (the IR-radio correlation) in star-forming galaxies established with IRAS is puzzling because of the many physical terms that connect IR to radio emission (Helou, Soifer \& Rowan-Robinson 1985; Condon 1992). Spitzer's sensitivity permits this ratio to be measured at higher redshifts and to be explored in spatial detail within nearby galaxies, both pursuits aimed at more insight into its origins. The 
correlation holds with its local value out to the redshifts $z \sim 1$ based on using 8 - and $24-\mu \mathrm{m}$ fluxes to estimate far-IR luminosity (Appleton et al. 2004), but may start deviating toward IR excess for fainter, submilli-Jansky 24- $\mu$ m sources (Boyle et al. 2007).

Murphy et al. (2006a) demonstrated that the radio images of disk galaxies are best described as smeared versions of the IR images, with the smearing scale length reflecting the spread of cosmic ray electrons from star-forming regions where they are first accelerated. Murphy et al. (2006b, 2008) show that the smearing scale length depends on the intensity of star formation in the disk and relate it to the time elapsed since the most recent star-formation episode rather than variations in the parameters constraining diffusion and decay of the cosmic rays in the disk.

Bai et al. (2007) find no significant modification of the IR luminosity function in the Coma Cluster from that observed in the field, suggesting that even a $40 \times$ increase in galaxy density does not affect the global star-formation behavior of galaxy populations. However, E.J. Murphy, J.D.P. Kenney, G. Helou, A. Chung, and J.H. Howell (submitted) demonstrate that the radio disks of galaxies in the Virgo Cluster are significantly disturbed with respect to the IR disks. The disturbances, presumably due to ram pressure by the intracluster medium, may be described as a partial displacement or ablation of the cosmic-ray plasma. Moreover, the radio surface brightness is abnormally elevated with respect to the IR brightness, an effect attributed to energy deposited in the plasma by interaction with the intracluster medium. These radio-to-IR comparisons illuminate for the first time the large-scale behavior of the cosmic ray plasma in external galaxies.

Abnormally high IR-to-radio ratios are very rare among galaxies, but they suggest "nascent starbursts," galaxies within a few million years of the onset of a starburst after a quiescence long enough to have dissipated the radio-emitting cosmic-ray electrons. Roussel et al. (2006) studied NGC 1377 as a prototype of that class, revealing a peculiar IR SED pointing to a compact, opaque source dominated by a hot continuum peaking at $\sim 30 \mu \mathrm{m}$ with $\tau \sim 20$ in the $10-\mu \mathrm{m}$ silicate band. The most prominent lines are $\mathrm{H}_{2}$ rotational transitions, presumably excited by shocks. The favored interpretation is that the young HII region is dense and dusty, with $85 \%$ of ionizing photons absorbed by dust.

Engelbracht et al. (2006) used broad-band images and spectra to study dust and aromatic feature emission in the superwind from the starburst in M82 out to $6 \mathrm{kpc}$ along the minor axis, and elsewhere in the halo of the galaxy, providing striking evidence of dust surviving energetic wind conditions and enriching the intergalactic medium.

Markwick-Kemper et al. (2007) present a rich dust-emission spectrum for the BAL quasar PG2112+059. In contrast to the quasars discussed earlier, which have spectra reasonably well fit by standard interstellar grain materials, the PG2112+059 spectrum shows unusual mineralogy in the form of corundum- $\mathrm{Al}_{2} \mathrm{O}_{3}$-also seen in the spectra of mass-losing AGB stars in the galaxy. This and other features of the spectrum led Markwick-Kemper et al. to propose that the grains in this case are formed in the cooling, expanding wind that also produces the broad absorption lines. Their results require the wind to have an inhomogeneous density and temperature structure, which is in agreement with some previous analyses. In addition, Markwick-Kemper et al. propose that dust formation in quasar winds may account for the dust seen in quasars at $z \sim 6$, an epoch too early in the life of the universe for dust to be attributable to formation in AGB winds.

\subsection{Summary}

Spitzer's imaging studies of nearby galaxies return striking images and reveal spatially detailed spectra of galaxies, including a hot dust component at $3-5 \mu \mathrm{m}$, and $8-\mu \mathrm{m}$ aromatic feature emission surrounding the more compact $24-\mu \mathrm{m}$ emission from HII regions. The SEDs are well fit by models with dust spread over a range of heating intensities; they show no need for $\mathrm{T}<10 \mathrm{~K}$ dust. The 
aromatic fraction drops quickly below $\sim 1 / 4$ solar metallicity. Spectroscopic studies show that the variations in the shape and intensity of mid-IR aromatic features relative to far-IR dust emission are driven by the presence of an AGN or variations in metallicity; the SEDs of irregular dwarf galaxies vary much more widely than among spirals, presumably because of their smaller size and greater volatility.

Studies of local ULIRGs demonstrate the power of IR spectroscopy to estimate the luminosity contribution from AGN based on the fluxes of high excitation lines. Wide variations in silicate absorption and aromatic emission invalidate the notion of a "typical" ULIRG spectrum, in spite of common features such as the fine-structure lines and frequent absorption by water or other ices, by crystalline silicates, and by molecules found in the dense ISM. Progress continues in distinguishing between the signatures of an AGN presence and large optical depth in ULIRG spectra.

Faint, red sources from 24- $\mu$ m surveys yield ULIRGs at $z \sim 1$ to $>3$, in spite of the uncertain estimates of their bolometric luminosity. Spectroscopic follow-up reveals as many as a third showing aromatic features, and at least a similar fraction with strong silicate absorption; these statistics vary with sample criteria. Using spectral attributes to ascertain the power source, AGN are generally found to prevail at $>3 \times 10^{12} L_{\odot}$, with pure starbursts occasionally reaching into this luminosity range. In an emerging contrast, $24-\mu \mathrm{m}$-selected samples are richer in AGN whereas submillimeter-selected samples favor star formation as the powering mechanism.

Silicate emission is detected for the first time in QSOs, and in combination with the silicate absorption data this has strengthened the unified model of Type I and Type II AGN, which invokes the observer's vantage point with respect to the dust torus in order to explain type differences. High-excitation line fluxes and dust SEDs suggest that QSOs can be surrounded by ULIRG-class starbursts.

The rotational lines of $\mathrm{H}_{2}$ are detected in every type of extragalactic object from dwarf galaxies to AGNs and are found to carry a fraction of the total dust luminosity that varies with object type. They are clearly enhanced by shocks and carry a striking $30 \%$ of cooling in some intergalactic shocks.

A powerful new estimator of SFR combining $24 \mu \mathrm{m}$ and $\mathrm{H} \alpha$ fluxes is used to explore the spatially resolved Schmidt law of star formation, which was found to be consistent with the global law. Irregular galaxies are clumpier, warmer, and have higher UV/IR ratios than spirals, as one would expect from a more porous ISM.

The radio images of galaxies look like smeared IR images, with the scale length of smearing related to the brightness of the disk; the scale length reflects the diffusion of the cosmic ray plasma. In the Virgo cluster, ram pressure seems to ablate the cosmic-ray plasma, resulting in an offset between the radio and IR images, while at the same time elevating the synchrotron brightness of the cosmic rays.

\section{SUMMARY AND LOOKING TO THE FUTURE}

The Spitzer Space Telescope is setting a new standard for studying the extragalactic universe at IR wavelengths. Although building on the tremendously successful IRAS and ISO missions, Spitzer's enormous gain in sensitivity has enabled studies of objects literally at the farthest reaches of the universe, has discovered new classes of objects and revealed shifts in galaxy populations at intermediate redshifts, and has yielded insights into the physics of interstellar processes in a variety of environments that drive or modulate galaxy evolution. As we write this review, there are $\sim 15$ months of the Spitzer cryogenic mission left. We expect more amazing results to emerge, adding to the harvest of the first four years of the mission. 
The most important findings so far from the Spitzer extragalactic studies include the following:

- Massive galaxies have been found less than a billion years after the Big Bang. This is a great surprise and challenges current ideas about galaxy formation.

- Sources detected in Spitzer surveys at $\lambda>20 \mu \mathrm{m}$, either via direct detections or statistical coadditions, appear to account for the far-IR background radiation out to $\sim 200 \mu \mathrm{m}$. Studies of the origin of the luminosity in far-IR sources suggest that $10-20 \%$ of the background radiation is attributable to AGN; the rest is attributed to dust-enshrouded star formation.

- The direct detection of dust-enshrouded star formation allows a measurement of the total SFR in the universe as a function of redshift out to $z>3$. This validates prior studies that applied significant corrections for dust extinction in the distant galaxies.

- The number counts of far-IR sources show strong evolution in the population of far-IR emitters as a function of redshift. This reflects the increase in dust-enshrouded SFR with redshift and reveals a shift in the IR luminosity function of galaxies toward LIRG dominance and ULIRG prominence at $z \sim 1-2$.

- At $z \sim 2,24-\mu \mathrm{m}$-selected samples are rich in embedded AGN and mid-IR enhanced SEDs. This contrasts with submillimeter surveys, which favor star formation as a power source and cooler, aromatics-rich SEDs.

- A population of 24- $\mu \mathrm{m}$ bright sources at redshifts between 1.5 and 2.5 has been identified as heavily obscured or even Compton-thick AGN. This population is perhaps twice as abundant as X-ray detected AGN at these redshifts.

- Aromatic features have been found in galaxy spectra for systems at redshifts up to $\sim 3$. This indicates that the processes that populate the ISM with carbon-rich materials were well in place $\sim 2$ Gyr after the Big Bang.

- Aromatic features, commonplace in gas-rich galaxies, appear to diminish in strength rapidly for galaxies with metallicity $<\sim 1 / 4$ solar. This implies that $z \sim 2$ ULIRGs with aromaticto-dust ratios similar to today's galaxies already had their ISM at close to solar metallicity.

- Silicate emission is found in quasar spectra. This provides another crucial link to the dustenshrouded AGN, consistent with unified models for AGN.

- Strong molecular hydrogen emission in pure rotational lines is detected in a wide range of systems ranging from dwarfs to normal galaxies to AGN and ULIRGs. These lines carry from $\sim 3 \times 10^{-5}$ to $\sim 3 \times 10^{-3}$ of the IR luminosity from dust. In some cases of intergalactic shocks, molecular $\mathrm{H}$ can account for as much as $30 \%$ of the total luminosity of a system, demonstrating the potential of $\mathrm{H}_{2}$ as a critical cooling channel in early-universe star formation.

- Spitzer 24- $\mu \mathrm{m}$ and $\mathrm{H} \alpha$ data have been combined linearly into more accurate star-formation measures than previously available; the improved measures have been used to extend the Schmidt law of star formation within disks of galaxies.

These highlights represent our subjective selection and are but a small fraction of the extragalactic work that Spitzer has accomplished to date. Moreover, as indicated in several sections above, advances on some key questions await more detailed analysis of existing data. Beyond the questions already being pursued, the Spitzer data archive offers a tremendous reservoir of potential results from ancillary or serendipitous science, or from recursive investigations building on first-round results. Archival research should thus yield a steady stream of results for years to come, as has been the case for rich astronomical archives such as IRAS and Hubble.

How will IR studies of the universe (particularly at wavelengths beyond $2 \mu \mathrm{m}$ ) advance from here? After the cryogenic mission ends, Spitzer will continue to have full sensitivity in the IRAC 3.6- and 4.5- $\mu \mathrm{m}$ channels. A five-year "warm Spitzer mission" utilizing these wavelengths will 
provide unmatched capabilities, and offer the opportunity, through large observing programs ( $>1000$ hrs/program), to make vital advances. Two examples of this are studies of massive galaxies at $z>6$, where a confusion-limited $(\sim 0.1 \mu \mathrm{Jy})$ survey of several square degrees could reveal thousands of galaxies at $6<z<8$, and a survey of several hundred square degrees to the depth of $1-2 \mu \mathrm{Jy}$ at $3.6 \mu \mathrm{m}$, which could identify bright quasars at $z>8$, hundreds of massive clusters of galaxies at $z>1$, and many tens of such clusters at $z>1$.5. Such studies, requiring 3000-5000 observing hours each, would have a profound impact on our understanding of the formation and evolution of structures in the universe.

There are many current observatories and future space- and ground-based facilities that will actively pursue and extend the discoveries from Spitzer. Here we mention two space observatories that will follow most directly on the Spitzer work. The Herschel Space Observatory, to be launched in late 2008, with imaging and spectroscopic capabilities at wavelengths longward of $70 \mu \mathrm{m}$ and a 3.5-m aperture, will be able to reach substantially deeper at long wavelengths where Spitzer is limited by confusion. In addition, the wavelength coverage of Herschel to well beyond $200 \mu \mathrm{m}$ will enable direct measurements of bolometric luminosities of high-redshift objects where Spitzer's $24-\mu \mathrm{m}$ observations sample rest-frame emission at $\lambda<10 \mu \mathrm{m}$. In addition, the long-wavelength spectroscopic capabilities of Herschel will provide new insights into the physical conditions in very dusty systems.

The Fames Webb Space Telescope, with a 50-Kelvin telescope and 60 times the collecting area of Spitzer, will far surpass the sensitivity and angular resolution of Spitzer in the mid-IR when it is launched in the next decade. JWST will enable more detailed studies of the distant universe revealed by Spitzer. Its imaging and spectroscopy at $\lambda<25 \mu \mathrm{m}$ will provide powerful probes of sources that Spitzer can detect only in deep imaging. This will be another suitably impressive follow-on mission to Spitzer.

The discoveries of Spitzer bear little resemblance to what was envisioned at the time the mission was defined. We look forward with great enthusiasm to the remainder of the Spitzer mission and its continuing revelations about the universe.

\section{DISCLOSURE STATEMENT}

The authors are not aware of any biases that might be perceived as affecting the objectivity of this review.

\section{ACKNOWLEDGMENTS}

We wish to acknowledge all the people whose work we have been able to cite, as well as those researchers whose work we were unable to cite because of lack of space, and we thank many members of the Spitzer community for providing suggestions and preprints. It is especially appropriate to acknowledge the instrument teams whose beautiful handiwork has led to the spectacular results we have described-the IRAC team led by Giovanni Fazio, the IRS team led by James Houck, and the MIPS team led by George Rieke. We thank E. van Dishoeck, B. Brandl, R. Genzel, G. Rieke, and G. Neugebauer for commenting on the manuscript. We also thank Marilyn Morgan, Mary Young, and Jim Jackson for editorial assistance, and Caroline Bot for assistance in preparing Figure 13. This work was supported by the National Aeronautics and Space Administration (NASA). Part of this work was carried out at the Jet Propulsion Laboratory, California Institute of Technology, under a contract with NASA. 


\section{LITERATURE CITED}

Alonso-Herrero A, Rieke GH, Rieke MJ, Colina L, Pérez-González PG, Ryder SD. 2006. Ap. 7. 650:835-49 Appleton PN, Fadda DT, Marleau FR, Frayer DT, Helou G, et al. 2004. Ap. F. Suppl. 154:147-50 Appleton PN, Xu KC, Reach W, Dopita MA, Gao Y, et al. 2006. Ap. 7. Lett. 639:L51-54 Armus L, Bernard-Salas J, Spoon HWW, Marshall JA, Charmandaris V, et al. 2006. Ap. F. 640:204-10 Armus L, Charmandaris V, Bernard-Salas J, Spoon HWW, Marshall JA, et al. 2007. Ap. F. 656:148-67 Babbedge TSR, Rowan-Robinson M, Vaccari M, Surace JA, Lonsdale CJ, et al. 2006. MNRAS 370:1159-80 Bai L, Marcillac D, Rieke GH, Rieke MJ, Tran K-V, et al. 2007. Ap. F. 664:181-97 Ballantyne DR, Papovich C. 2007. Ap. 7. 660:988-94 Barger AJ, Cowie LL, Mushotzky RF, Yang Y, Wang W-H, et al. 2005. Ap. 7. 129:578-609 Barmby P, Ashby MLN, Bianchi L, Engelbracht CW, Gehrz RD, et al. 2006. Ap. 7. Lett. 650:L45-49 Bell EF, Zheng XZ, Papovich C, Borch A, Wolf C, Meisenheimer K. 2007. Ap. 7. 663:834-43 Bendo GJ, Buckalew BA, Dale DA, Draine BT, Joseph RB, et al. 2006. Ap. 7. 645:134-47 Blain AW, Small I, Ivison RJ, Kneib J-P, Frayer DT. 2002. Phys. Rev. 369:111-76 Bolatto AD, Simon JD, Stanimirovic S, van Loon JT, Shah RY, et al. 2007. Ap. 7. 655:212-32 Borys C, Blain AW, Dey A, Le Floc'h E, Jannuzi BT, et al. 2006. Ap. 7. 636:134-39 Bouwens RJ, Illingworth GD, Blakeslee J, Franx M. 2006. Ap. 7. 653:53-85 Bouwens RJ, Illingworth GD, Thompson RI, Franx M. 2005. Ap. F. Lett. 624:L5-8 Bouwens RJ, Thompson RI, Illingworth GD, Franx M, von Dokkum PG, et al. 2004. Ap. F. Lett. 616:L79-82 Boyle BJ, Cornwell TJ, Middelberg E, Norris RP, Appleton PN, Smail I. 2007. MNRAS 376:1182-88 Brand K, Dey A, Desai V, Soifer BT, Bian C, et al. 2007. Ap. F. 663:204-17 Brand K, Dey A, Weedman D, Desai V, Le Floc'h E, et al. 2006. Ap. 7. 644:143-47 Brandl B, Bernard-Salas J, Spoon HWW, Devost D, Sloan GC, et al. 2006. Ap. 7. 653:1129-44 Bressan A, Panuzzo P, Buson L, Clemens M, Granato GL, et al. 2006. Ap. F. Lett. 639:55-58 Brunner G, Sheth K, Armus L, Wolfire M, Vogel S, et al. 2008. Ap. 7. 675:316-329 Bunker AJ, Stanway ER, Ellis RS, McMahon RG. 2004. MNRAS 355:374-84 Calzetti D, Kennicutt RC, Engelbracht CW, Leitherer C, Draine BT, et al. 2007. Ap. F. 666:870-95 Caputi KI, Dole H, Lagache G, McLure RJ, Dunlop JS, et al. 2006. Astron. Astrophys. 454:143-50 Caputi KI, Lagache G, Yan L, Dole H, Bavouzet N, et al. 2007. Ap. 7. 660:97-116 Chabrier G. 2003. Publ. Astron. Soc. Pac. 115:763-95

Chary R, Casertano S, Dickinson ME, Ferguson HC, Eisenhardt PRM, et al. 2004. Ap. F. Suppl. 154:80-86 Chary R, Elbaz D. 2001. Ap. 7. 556:562-81

Chary R, Stern D, Eisenhardt P. 2005. Ap. 7. Lett. 635:L5-8

Chary R, Teplitz HI, Dickinson ME, Koo DC, Le Floc'h E, et al. 2007. Ap. 7. 665:257-64

Churchwell E, Povich MS, Allen D, Taylor MG, Meade MR, et al. 2006. Astron. 7. 649:759-78

Cole S, Norberg P, Baugh CM, Frenk CS, Bland-Hawthorn J, et al. 2001. MNRAS 326:255-73

Condon JJ. 1992. Annu. Rev. Astron. Astrophys. 30:575-611

Cooray A, Sullivan I, Chary R, Bock JJ, Dickinson M, et al. 2007. Ap. 7. Lett. 659:L91-94

Daddi E, Alexander DM, Dickinson M, Gilli R, Renzini A, et al. 2007a. Ap. 7. 670:173-89

Daddi E, Cimatii A, Renzini A, Fontana A, Mignoli M. 2004. Ap. 7. 617:746-64

Daddi E, Dickinson M, Chary R, Pope A, Morrison G, et al. 2005. Ap. 7. Lett. 631:L13-16

Daddi E, Dickinson M, Morrison G, Chary R, Cimatti A, et al. 2007b. Ap. F. 670:156-72

Dale DA, Bendo GJ, Engelbracht CW, Gordon KD, Regan MW, et al. 2005. Ap. F. 633:857-70

Dale DA, Gil de Paz A, Gordon KD, Hanson HM, Armus L, et al. 2007. Ap. 7. 655:863-84

Dale DA, Helou G. 2002. Ap. 7. 576:159-68

Dale DA, Helou G, Contursi A, Silbermann NA, Kolhatkar S. 2001. Ap. 7. 549:215-27

Dale DA, Smith JDT, Armus L, Buckalew BA, Helou G, et al. 2006. Ap. F. 646:161-73

De Propris R, Stanford SA, Eisenhardt PR, Holden BP, Rosati P. 2007. Ap. F. 133:2209-15

Desai V, Armus LL, Spoon HWW, Charmandaris V, Bernard-Salas J, et al. 2007. Ap. F. 669:810-20

Desai V, Soifer BT, Dey A, Jannuzi BT, Le Floc'h E, et al. 2008. Ap. 7. 679:1204-17 
Désert F-X, Boulanger F, Puget JL. 1990. Astron. Astrophys. 237:215-36

Dey A, Soifer BT, Desai V, Brand K, Le Floc'h E, et al. 2008. Ap. F. 677(No. 2):943-57

Dole H, Lagache G, Puget J-L, Caputi KI, Fernández-Conde N, et al. 2006. Astron. Astrophys. 451:417-29

Dole H, Rieke GH, Lagache G, Puget J-L, Alonso-Herrero A, et al. 2004. Ap. F. Suppl. 154:93-96

Draine BT, Dale DA, Bendo G, Gordon KD, Smith JDT, et al. 2007. Ap. 7. 663:866-94

Dunlop JS, Cirasuolo M, McLure RJ. 2007. MNRAS 376:1054-64

Dye S, Eales SA, Ashby MLN, Huang J-S, Egami E, et al. 2007. MNRAS 375:725-34

Egami E, Kneib J-P, Rieke GH, Ellis RS, Richard J, et al. 2005. Ap. 7. Lett. 618:L5-8

Egami E, Rieke GH, Fadda D, Hines DC. 2006. Ap. F. Lett. 652:L21-24

Elbaz D. 2005. Space Sci. Rev. 119:93-119

Elbaz D, Cesarsky CJ, Chanial P, Aussel H, Franceschini A, et al. 2002. Astron. Astrophys. 384:848-65

Elmegreen DM, Elmegreen BG, Kaufman M, Sheth K, Struck C, et al. 2006. Ap. 7. 642:158-70

Engelbracht CW, Gordon KD, Rieke GH, Werner MW, Dale DA, Latter WB. 2005. Ap. F. Lett. 628:L29-32

Engelbracht CW, Kundurthy P, Gordon KD, Rieke GH, Kennicut RC, et al. 2006. Ap. F. Lett. 642:L127-32

Erb DK, Shapley AE, Steidel CC, Pettini M, Adelberger KL, et al. 2003. Ap. 7. 591:101-18

Eyles LP, Bunker AJ, Ellis RS, Lacy M, Stanway ER, et al. 2007. MNRAS 374:910-30

Eyles LP, Bunker AJ, Stanway ER, Lacy M, Ellis RS, Doherty M. 2005. MNRAS 364:443-54

Farrah D, Bernard-Salas J, Spoon HWW, Soifer BT, Armus L, et al. 2007. Ap. 7. 667:149-69

Fazio GG, Ashby MLN, Barmby P, Hora JL, Huang J-S, et al. 2004. Ap. 7. Suppl. 154:39-43

Franx M, Labbé I, Rudnick G, van Dokkum PG, Daddi E, et al. 2003. Ap. 7. Lett. 587:L79-82

Frayer DT, Huynh MT, Chary R, Dickinson M, Elbaz D, et al. 2006. Ap. F. Lett. 647:L9-12

Gehrz RD, Roellig TL, Werner MW, Fazio GG, Houck JR, et al. 2007. Rev. Sci. Instrum. 78:011302-39

Genzel R, Cesarsky C. 2000. Annu. Rev. Astron. Astrophys. 38:761-814

Genzel R, Lutz D, Sturm E, Egami E, Kunze D, et al. 1998. Ap. 7. 498:579-605

Goldader JD, Meurer G, Heckman TM, Seibert M, Sanders DB, et al. 2002. Ap. F. 568:651-78

Gordon KD, Bailin J, Engelbracht CW, Rieke GH, Misselt KA, et al. 2006. Ap. F. Lett. 638:L87-92

Gruppioni C, Pozzi F, Lari C, Oliver S, Rodighiero G. 2005. Ap. 7. Lett. 618:L9-12

Hao L, Spoon HWW, Sloan GC, Marshall JA, Armus L, et al. 2005. Ap. 7. Lett. 625:L75-78

Hauser MG, Dwek E. 2001. Annu. Rev. Astron. Astrophys. 39:249-307

Heckman TM. 1994. In Mass-Transfer Induced Activity in Galaxies, ed. I Shlosman, pp. 234-50. Cambridge: Cambridge Univ. Press

Helou G, Roussel H, Appleton P, Frayer D, Stolovy S, et al. 2004. Ap. 7. Suppl. 154:253-58

Helou G, Soifer BT, Rowan-Robinson M. 1985. Ap. 7. Lett. 298:L7-11

Hernquist L, Springel V. 2003. MNRAS 341:1253-67

Higdon SJU, Armus L, Higdon JL, Soifer BT, Spoon HWW. 2006. Ap. 7. 648:323-39

Higdon SJU, Higdon JL, Marshall J. 2006. Ap. F. 640:768-83

Hinz JL, Misselt K, Rieke MJ, Rieke GH, Smith PS, et al. 2006. Ap. 7. 651:874-81

HinzJL, Rieke GH, Gordon KD, Pérez-González PG, Engelbracht CW, et al. 2004. Ap. 7. Suppl. 154:259-62

Hogg DW, Tremonti CA, Blanton MR, Finkbeiner DP, Padmanabhan N, et al. 2005. Ap. 7. 624:162-67

Houck JR, Charmandaris V, Brandl BR, Weedman D, Herter T, et al. 2004. Ap. F. Suppl. 154:211-14

Houck JR, Soifer BT, Weedman D, Higdon SJU, Higdon JL, et al. 2005. Ap. F. Lett. 622:L105-8

Hunter DA, Elmegreen BG, Martin E. 2006. Astron. F. 132:801-18

Huynh MT, Frayer DT, Mobasher B, Dickinson M, Chary R, Morrison G. 2007a. Ap. 7. Lett. 667:L9-12

Huynh MT, Pope A, Frayer DT, Scott D. 2007b. Ap. 7. 659:305-13

Imanishi M, Dudley CC, Maiolino R, Maloney PR, Nakagawa T, Risaliti G. 2007. Ap. F. Suppl. 171:72-100

Jackson DC, Cannon JM, Skillman ED, Lee H, Gehrz R, et al. 2006. Ap. F. 646:192-204

Jiang L, Fan X, Hines DC, Shi Y, Vestergaard M, et al. 2006. Astron. 7. 132:2127-34

Kashlinsky A, Arendt RG, Mather J, Moseley SH. 2005. Nature 438:45-50

Kashlinsky A, Arendt RG, Mather J, Moseley SH. 2007a. Ap. 7. Lett. 654:L1-4

Kashlinsky A, Arendt RG, Mather J, Moseley SH. 2007b. Ap. 7. Lett. 654:L5-8

Kennicutt RC. 1998. Ap. F. 498:541-52

Kennicutt RC Jr, Armus L, Bendo G, Calzetti D, Dale DA, et al. 2003. Publ. Astron. Soc. Pac. 115:928-52

Kennicutt RC Jr, Calzetti D, Walter F, Helou G, Hollenbach D, et al. 2007. Ap. 7. 67:333-348 
Kneib JP, Ellis RS, Santos MR, Richard J. 2004. Ap. f. 607:697-703

Kraemer KE, Shipman RF, Price SD, Mizuno DR, Kuchar T, Carey SJ. 2003. Astron. F. 126:1423-50

Kroupa P. 2001. MNRAS 322:231-46

Labbé I, Bouwens R, Illingworth GD, Franx M. 2006. Ap. 7. Lett. 649:L67-70

Lacy M, Petric AO, Sajina A, Canalizo G, Storrie-Lombardi LJ, et al. 2007. Astron. 7. 133:186-205

Lacy M, Storrie-Lombardi LJ, Sajina A, Appleton PN, Armus L, et al. 2004. Ap. 7. Suppl. 154:166-69

Lagache G, Bavouzet N, Fernandez-Conde N, Ponthieu N, Rodet T, et al. 2007. Ap. F. Lett. 665:L89-92

Lagache G, Dole H, Puget J-L. 2003. MNRAS 338:555-71

Lagache G, Dole H, Puget J-L, Pérez-González PG, Le Floc'h E, et al. 2004. Ap 7. Suppl. 154:112-17

Lagache G, Puget J-L, Dole H. 2005. Annu. Rev. Astron. Astrophys. 43:727-68

Lahuis F, Spoon HWW, Tielens AGGM, Doty SD, Armus L, et al. 2007. Ap. 7. 659:296-304

Lai K, Huang J-S, Fazio G, Cowie LL, Hu EM, Kakazu Y. 2007. Ap. 7. 655:704-13

Laurent O, Mirabel IF, Charmandaris V, Gallais P, Madden SC, et al. 2000. Astron. Astrophys. 359:887-99

Lee H, Skillman ED, Cannon JM, Jackson DC, Gehrz R, et al. 2006. Ap. 7. 647:970-83

Le Floc'h E, Papovich C, Dole H, Bell E, Lagache G, et al. 2005. Ap. 7. 632:169-90

Levenson NA, Sirocky MM, Hao L, Spoon HWW, Marshall JA, et al. 2007. Ap. F. Lett. 654:L45-48

Lilly SJ, Tresse L, Hammer F, Crampton D, Le Fevre O. 1995. Ap. 7. 455:108-24

Lonsdale CJ, Smith HE, Rowan-Robinson M, Surace J, Shupe D, et al. 2003. Publ. Astron. Soc. Pac. 115:897-927

Lutz D, Yan L, Armus L, Helou G, Tacconi LJ, et al. 2005. Ap. F. Lett. 632:L13-16

Madau P, Ferguson HC, Dickinson ME, Giavalisco M, Steidel CC, Fruchter A. 1996. MNRAS 283:1388-404

Malhotra S, Kaufman MJ, Hollenbach D, Helou G, Rubin RH, et al. 2001. Ap. 7. 562:766-86

Maloney PR, Hollenbach DJ, Tielens AGGM. 1996. Ap. 7. 466:561-84

Mannucci F, Buttery H, Maiolino R, Marconi A, Pozzetti L. 2007. Astron. Astrophys. 461:423-31

Maraston C. 2005. MNRAS 362:799-825

Maraston C, Daddi E, Renzini A, Cimatti A, Dickinson M, et al. 2006. Ap. F. 652:85-96

Marcillac D, Rigby JR, Rieke GH, Kelly DM. 2007. Ap. 7. 654:825-34

Markwick-Kemper F, Gallagher SC, Hines DC, Bouwman J. 2007. Ap. F. Lett. 668:L107-10

Marleau FR, Fadda D, Storrie-Lombardi LJ, Helou G, Makovoz D, et al. 2004. Ap. 7. Suppl. 154:66-69

Meixner M, Gordon KD, Indebetouw R, Hora JL, Whitney B, et al. 2006. Astron. F. 132:2268-88

Meurer G, Heckman TM, Calzetti D. 1999. Ap. 7. 521:64-80

Mobasher B, Dickinson M, Ferguson HC, Giavalisco M, Wiklind T, et al. 2005. Ap. 7. 635:832-44

Murphy EJ, Braun R, Helou G, Armus L, Kenney JDP, et al. 2006a. Ap. 7. 638:157-75

Murphy EJ, Helou G, Braun R, Kenney JDP, Armus L, et al. 2006b. Ap. F. Lett. 651:L111-15

Murphy EJ, Helou G, Kenney JDP, Armus L, Braun R. 2008. Ap. F. 678:828-50

Netzer H, Lutz D, Schweitzer M, Contursi A, Sturm E, et al. 2007. Ap. 7. 666:806-16

Noeske KG, Faber SM, Weiner BJ, Koo DC, Primack JR, et al. 2007a. Ap. 7. Lett. 660:L47-50

Noeske KG, Weiner BJ, Faber SM, Papovich C, Koo DC, et al. 2007b. Ap. 7. Lett. 660:L43-46

Ogle P, Antonucci R, Appleton PN, Whysong D. 2007. Ap. F. 668:699-707

O'Halloran B, Satyapal S, Dudik RP. 2006. Ap. F. 641:795-800

Pahre MA, Ashby MLN, Fazio GG, Willner SP. 2004a. Ap. F. Suppl. 154:229-34

Pahre MA, Ashby MLN, Fazio GG, Willner SP. 2004b. Ap. F. Suppl. 154:235-41

Papovich C, Dole H, Egami E, Le Floc'h E, Pérez-González PG, et al. 2004. Ap. 7. Suppl. 154:70-74

Papovich C, Moustakas LA, Dickinson M, Le Floc'h E, Rieke GH, et al. 2006. Ap. 7. 640:92-113

Papovich C, Rudnick G, Le Floc'h E, von Dokkum PG, Rieke GH, et al. 2007. Ap. F. 668:45-61

Pérez-González PG, Rieke GH, Egami E, Alonso-Herrero A, Dole H, et al. 2005. Ap. 7. 630:82-107

Pope A, Chary R, Dickinson M, Scott S. 2008. In ASP Conf. Ser. 381:249-54

Pope A, Scott D, Dickinson M, Chary R, Morrison G, et al. 2006. MNRAS 370:1185-207

Reddy NA, Steidel CC, Fadda D, Yan L, Pettini M, et al. 2006. Ap. F. 644:792-812

Reddy NA, Steidel CC, Pettini M, Adelberger KL, Shapley AE, et al. 2008. Ap. 7. Suppl. 175(No. 1):48-85

Richards GT, Lacy M, Storrie-Lombardi LJ, Hall PB, Gallagher SC, et al. 2006. Ap. F. Suppl. 166:470-97

Rieke GH, Low FJ. 1972. Ap. F. Lett. 176:L95-100

Rigby JR, Marcillac D, Egami E, Rieke GH, Richard J, et al. 2008. Ap. 7. 675:262-80

Rodighiero G, Ciamatti A, Franceschini A, Brusa M, Fritz J, Bolzonella M. 2007. Astron. Astrophys. 470:21-37 
Rodighiero G, Lari C, Pozzi F, Gruppioni C, Fadda D. 2006. MNRAS 371:1891-97

Rosenberg JL, Ashby MLN, Salzer JJ, Huang J-S. 2006. Ap. 7. 636:742-52

Roussel H, Helou G, Smith JD, Draine BT, Hollenbach DJ, et al. 2006. Ap. 7. 646:841-57

Roussel H, Helou G, Hollenbach DJ, Draine BT, Smith JD, et al. 2007. Ap. F. Suppl. 669:959-81

Sajina A, Yan L, Armus L, Choi P, Fadda D, et al. 2007. Ap. 7. 664:713-37

Sanders DB, Mazzarella JM, Kim D-C, Surace JA, Soifer BT. 2003. Astron. 7. 126:1607-64

Sanders DB, Mirabel IF. 1996. Annu. Rev. Astron. Astrophys. 34:749-92

Sanders DB, Salvato M, Aussel H, Ilbert O, Scoville N, et al. 2007. Ap. 7. Suppl. 172:86-98

Sanders DB, Soifer BT, Elias JH, Madore BF, Matthews K, et al. 1988. Ap. 7. 325:74-91

Sauvage M, Tuffs RJ, Popescu CC. 2005. Space Sci. Rev. 119:313-53

Schweitzer M, Lutz D, Sturm E, Contursi A, Tacconi LJ, et al. 2006. Ap. 7. 649:79-90

Shapley AE, Steidel CC, Erb DK, Reddy NA, Adelberger KL, et al. 2005. Ap. F. 626:698-722

Shi Y, Ogle P, Rieke GH, Antonucci R, Hines DC, et al. 2007. Ap. 7. 669:841-61

Shi Y, Rieke GH, Hines DC, Gorjian V, Werner MW, et al. 2006. Ap. 7. 653:127-36

Siebenmorgen R, Haas M, Krügel E, Schulz B. 2005. Astron. Astrophys. Lett. 436:L5-8

Smith JDT, Draine BT, Dale DA, Moustakas J, Kennicutt RC Jr, et al. 2007. Ap. 7. 656:770-91

Soifer BT, Helou G, Lonsdale CJ, Neugebauer G, Hacking P, et al. 1984. Ap. 7. Lett. 283:L1-4

Soifer BT, Houck JR, Neugebauer G. 1987. Annu. Rev. Astron. Astrophys. 25:187-230

Soifer BT, Neugebauer G, Matthews K, Egami E, Becklin EE, et al. 2000. Astron. 7. 119:509-23

Spoon HWW, Marshall JA, Houck JR, Elitzur M, Hao L, et al. 2007. Ap. 7. Lett. 654:L49-52

Spoon HWW, Tielens AGGM, Armus L, Sloan GC, Sargent B, et al. 2006. Ap. 7. 638:759-65

Stark DP, Bunker AJ, Ellis RS, Eyles LP, Lacy M. 2007. Ap. 7. 659:84-97

Steidel CC, Adelberger KL, Giavalisco M, Dickinson M, Pettini M. 1999. Ap. 7. 519:1-17

Steidel CC, Giavalisco M, Pettini M, Dickinson M, Adelberger KL. 1996. Ap. F. Lett. 462:L17-21

Steidel CC, Hamilton D. 1993. Astron. 7. 105:2017-30

Stern D, Eisenhardt P, Gorjian V, Kochanek S, Caldwell N, et al. 2005. Ap. F. 631:163-68

Sullivan I, Cooray A, Chary R, Bock JJ, Brodwin M, et al. 2007. Ap. F. 657:37-50

Teplitz HI, Desai V, Armus L, Chary R, Marshall JA, et al. 2007. Ap. 7. 659:941-49

Thuan TX, Sauvage M, Madden S. 1999. Ap. F. 516:783-87

Treister E, Megan Urry C, Van Duyne J, Dickinson M, Chary R-R, et al. 2006. Ap. 7. 640:603-11

Tyler KD, Le Floc'h E, Rieke GH, Dey A, Desai V, et al. 2008. Ap. F. In press

Valiante E, Lutz D, Sturm E, Genzel R, Tacconi LJ, et al. 2007. Ap. 7. 660:1060-71

Verma A, Charmandaris V, Klaas U, Lutz D, Haas M. 2005. Space Sci. Rev. 119:355-407

Walter F, Cannon JM, Roussel H, Bendo GJ, Calzetti D, et al. 2007. Ap. F. 661:102-14

Wang YP. 2006. Ap. 7. 638:138-47

Webb TMA, van Dokkum P, Egami E, Gazio G, Franx M, et al. 2006. Ap. F. Lett. 636:L17-20

Wen X-Q, Wu H, Cao C, Xia X-Y. 2007. Chin. 7. Astron. Astrophys. 7:187-98

Werner M, Fazio G, Rieke G, Roellig TL, Watson DM. 2006. Annu. Rev. Astron. Astrophys. 44:269-321

Wiklind T, Dickinson M, Ferguson HC, Giavalisco M, Mobasher B, et al. 2008. Ap. 7. 676:781-806

Wright EL, Reese ED. 2000. Ap. F. 545:43-55

Wu Y, Charmandaris V, Hunt LK, Bernard-Salas J, Brandl BR, et al. 2007. Ap. F. 662:952-58

Xu C, Lonsdale CJ, Shupe DL, O'Linger J, Masci F. 2001. Ap. 7. 562:179-207

Yan H, Dickinson M, Giavalisco M, Stern D, Eisenhardt PRM, Ferguson H. 2006. Ap. 7. 651:24-40

Yan H, Dickinson M, Stern D, Eisenhardt PRM, Chary R, et al. 2005a. Ap. 7. 634:109-27

Yan H, Windhorst R. 2004. Ap. 7. Lett. 600:L1-5

Yan L, Chary R, Armus L, Teplitz H, Helou G, et al. 2005b. Ap. 7. 628:604-10

Yan L, Sajina A, Fadda D, Choi P, Armus L, et al. 2007. Ap. 7. 658:778-93

Younger JD, Fazio GG, Huang J-S, Yun MS, Wilson GW, et al. 2007. Ap. F. 671:1531-37

Zheng XZ, Bell EF, Papovich C, Wolf C, Meisenheimer K, et al. 2007. Ap. F. Lett. 661:L41-44

Zheng XZ, Bell EF, Rix H-W, Papovich C, Le Floc'h E, et al. 2006. Ap. F. 640:784-800 


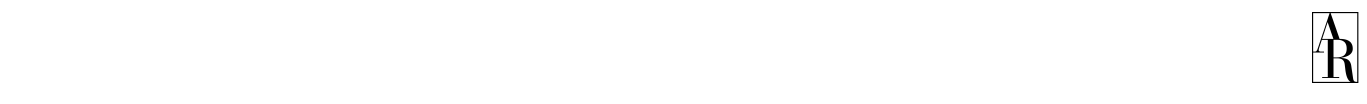

Annual Review of Astronomy and Astrophysics

\section{Contents}

A Serendipitous Journey

Alexander Dalgarno

The Growth Mechanisms of Macroscopic Bodies

in Protoplanetary Disks

Fürgen Blum and Gerhard Wurm

Water in the Solar System

Thérèse Encrenaz

Supernova Remnants at High Energy

Stephen P. Reynolds

The Crab Nebula: An Astrophysical Chimera

7. Feff Hester

Pulsating White Dwarf Stars and Precision Asteroseismology

D.E. Winget and S.O. Kepler

The Spitzer View of the Extragalactic Universe

Baruch T. Soifer, George Helou, and Michael Werner

Neutron-Capture Elements in the Early Galaxy

Christopher Sneden, Fohn 7. Cowan, and Roberto Gallino

Interstellar Polycyclic Aromatic Hydrocarbon Molecules

A.G.G.M. Tielens

Evolution of Debris Disks

Mark C. Wyatt

Dark Energy and the Accelerating Universe

Foshua A. Frieman, Michael S. Turner, and Dragan Huterer

Spectropolarimetry of Supernovae

Lifan Wang and 7. Craig Wheeler

Nuclear Activity in Nearby Galaxies

Luis C. Ho 
The Double Pulsar

M. Kramer and I.H. Stairs

\section{Indexes}

Cumulative Index of Contributing Authors, Volumes 35-46 ..................... 573

Cumulative Index of Chapter Titles, Volumes 35-46 .......................... 576

\section{Errata}

An online log of corrections to Annual Review of Astronomy and Astrophysics articles may be found at http://astro.annualreviews.org/errata.shtml 